TRANSACTIONS OF THE

AMERICAN MATHEMATICAL SOCIETY

Volume 351, Number 8, Pages 3387-3412

S 0002-9947(99)02363-6

Article electronically published on April 12, 1999

\title{
INVARIANT MEASURES FOR ALGEBRAIC ACTIONS, ZARISKI DENSE SUBGROUPS AND KAZHDAN'S PROPERTY $(T)$
}

\author{
YEHUDA SHALOM
}

\begin{abstract}
Let $k$ be any locally compact non-discrete field. We show that finite invariant measures for $k$-algebraic actions are obtained only via actions of compact groups. This extends both Borel's density and fixed point theorems over local fields (for semisimple/solvable groups, resp.). We then prove that for $k$-algebraic actions, finitely additive finite invariant measures are obtained only via actions of amenable groups. This gives a new criterion for Zariski density of subgroups and is shown to have representation theoretic applications. The main one is to Kazhdan's property $(T)$ for algebraic groups, which we investigate and strengthen.
\end{abstract}

\section{Introduction and Discussion of the Main Results}

In this paper we discuss measure theoretic aspects of regular actions of linear algebraic groups on algebraic varieties, where all the groups, homomorphisms, varieties and actions, are assumed to be defined over a fixed locally compact nondiscrete field $k$. Let $G$ be a linear $k$-algebraic group acting $k$-regularly on a $k$-variety $V$. If $G_{k}$ is compact, it admits a finite Haar measure, and so any $G_{k}$-orbit in $V_{k}$ will support a $G_{k}$-invariant probability measure. In the present paper we shall see that for regular actions of all linear algebraic groups, every invariant probability measure comes about via this construction, that is, the action must factor through a compact algebraic quotient. More precisely, our first main result, proved in Section 3 , is the following:

1.1 Theorem (No restriction on char $k$ ). For every $k$-group $G$ there exists a $k$ group $H$ which is $k$-compact (i.e. $H_{k}$ is compact), and a (separable) surjective $k$-homomorphism $\varphi: G \rightarrow H$ with the following property:

If $G$ acts $k$-regularly on a $k$-variety $V$, and $\mu$ is a $G_{k}$-invariant $\sigma$-additive probability measure on $V_{k}$, then there exists a $G$-invariant $k$-subvariety $W \subseteq V$ such that $\mu\left(W_{k}\right)=1$, and the action of $G$ on $W$ factors through $H$ via $\varphi$.

In particular, if the trivial group is the only $k$-image of $G$ which is $k$-compact, then every invariant measure is supported on fixed points.

Let us observe some applications of this result. Recall that the Borel density theorem [3] asserts that if $G_{k}$ is semisimple with no compact factors and $\Gamma<G_{k}$

Received by the editors March 26, 1997.

1991 Mathematics Subject Classification. Primary 14L30, 20G05, 22E50, 28 D15.

Partially sponsored by the Edmund Landau Center for research in Mathematical Analysis, supported by the Minerva Foundation (Germany). 
is a lattice, then $\Gamma$ is Zariski dense in $G$. This well known theorem has proven a fundamental tool in the ergodic theory of algebraic groups, and several authors have given other proofs and extensions of it (see, for example, [9], [10], [20], [27], [28], [35] and the references therein). From 1.1 one readily deduces:

1.2 Corollary. The Borel density theorem holds for $G$ (i.e. all lattices in $G_{k}$ are Zariski dense) iff every $k$-compact quotient of $G$ is trivial.

Indeed, to verify 1.2 simply notice that if $G$ satisfies the latter condition, and $\Gamma<G_{k}$ is a lattice with $H$ being its Zariski closure, then $H$ is defined over $k$, $\Gamma<H_{k}$, and we have the natural $G_{k}$-maps: $G_{k} / \Gamma \rightarrow G_{k} / H_{k} \hookrightarrow(G / H)_{k}$. Pushing forward the measure we obtain an invariant measure for the $G_{k}$-action on $V_{k}$, where $V=G / H$. By 1.1 there is a fixed point for the action of $G$ on $G / H$, hence $G=H$. The converse is obvious using the (finite) Haar measure on any compact quotient.

On the other hand, Theorem 1.1 also implies an analogue of Borel's fixed point theorem, over local fields (cf. [7, §9], when $\operatorname{char} k=0$ ):

1.3 Corollary. Let $G$ be a solvable k-group. Then the following are equivalent:

1. G has no non-trivial k-compact image.

2. If $V$ is a k-variety on which $G$ acts $k$-regularly, and $F \subseteq V_{k}$ is a non-empty compact $G_{k}$-invariant subset, then there is a $G$-fixed point in $F$.

Indeed, 1 implies 2 since $G_{k}$ being solvable, is amenable, so there exists an invariant measure for its action on $F$, and by 1.1 there is a fixed point there. To see the converse observe that if $\varphi: G \rightarrow H$ is a $k$-homomorphism, then $G$ acts on $H$ by left multiplication via $\varphi$. If $H_{k}$ is compact, by 2 there is a fixed point for this action, so $\varphi$ must be trivial.

Notice that when $k=\mathbb{C}$ condition 1 in 1.3 is automatically satisfied for every (algebraically) connected group. The notions "compactness" and "completeness", which are analogous in the Hausdorff and Zariski topologies resp., coincide in that case, and thus 1.3 reduces to Borel's fixed point theorem for regular actions of solvable algebraic groups. If char $k=0$ condition 1 is equivalent to $G$ being triangulizable over $k$. A complete discussion on the equivalence of various (algebraic, topological, measure theoretic) properties of solvable $k$-groups is given in 3.6.

Our unified formulation of Borel's density theorem, Theorem 1.1 above, covers an accumulation of results of several authors (e.g. [1], [9], [27], [28]), but does not depend on them. In the proof we use the following structure theorem:

1.4 Proposition. Every $k$-group $G$ has a maximal $k$-subgroup $N$ with no nontrivial $k$-compact image. $N$ is maximal in the sense that it contains every $k$ subgroup of $G$ with this property. $N$ is characteristic for $k$-automorphisms of $G$, and in particular $N \triangleleft G$. Moreover, $G / N$ is $k$-compact. $N$ is called the k-discompact radical of $G$.

Naturally, $G / N$ is the "maximal" $k$-compact quotient, alluded to in Theorem 1.1, through which all the algebraic measure preserving actions of $G$ factor. In fact, an analogous structure theorem holds with "amenability" replacing "compactness":

1.5 Proposition. In 1.4 one can replace everywhere "compact" by "amenable". This defines the k-disamenable radical. (See 4.4 for a complete formulation.)

The parallelism between "amenability" and "compactness", as hinted in 1.41.5 , is in fact the main subject of Section 4 , where we consider finitely additive 
measures. We shall find in that section the same phenomenon taking place: for algebraic actions, finite invariant measures occur "only when they have to". Recall first that locally compact amenable groups are characterized by having an invariant finitely additive probability measure for their self action, and hence for every continuous action. We will show that in Theorem 1.1 one can replace " $\sigma$-additive measure" by "finitely additive measure" if, at the same time, "compact" is replaced by "amenable". Specifically, our second main result is:

1.6 Theorem. Assume char $k=0$. Then for every $k$-group $G$ there exists a $k$ group $H$ which is $k$-amenable (i.e. $H_{k}$ is amenable) and a surjective $k$-homomorphism $\varphi: G \rightarrow H$ with the following property: If $G$ acts $k$-regularly on a $k$ variety $V$, and $\mu$ is a $G_{k}$-invariant finitely-additive probability measure on $V_{k}$, then there exists a $G$-invariant $k$-subvariety $W \subseteq V$ such that $\mu\left(W_{k}\right)=1$, and the action of $G$ on $W$ factors through $H$ via $\varphi$.

In particular, if the trivial group is the only $k$-image of $G$ which is $k$-amenable, then every invariant measure is supported on fixed points.

Exactly the same argument as in the proof of 1.2 then shows:

1.7 Corollary. If every $k$-amenable quotient of $G$ is trivial, and $\Gamma<G_{k}$ is such that $G_{k} / \Gamma$ supports a $G_{k}$-invariant finitely additive probability measure, then $\Gamma$ is Zariski dense in $G$.

Semisimple groups with no compact factors satisfy the condition on $G$ in 1.7 , thus we establish a generalization of the density theorem proved by Stuck [35] (see also [21]). We shall indeed see that the subgroups discussed in [35] meet the condition on $\Gamma$ in 1.7 , and mention other natural equivalent characterizations of this property (see 4.18 below).

Theorem 1.1 shows that when $G$ has no $k$-compact quotients and its action on the $k$-variety $V$ has no fixed points, there is no $G_{k}$-invariant probability measure on $V_{k}$. Under the analogous stronger assumption on $G$, we can deduce from 1.6 a quantitative version of this result:

1.8 Corollary. Suppose that the k-group $G$ has no nontrivial k-amenable quotients, and that there is no fixed point in its action on the $k$-variety $V$. Then there exist $\varepsilon>0, g_{1}, \cdots, g_{n} \in G_{k}$ and real bounded (Borel-)measurable functions $f_{1}, \cdots, f_{n}: V_{k} \rightarrow \mathbb{R}$ such that the following holds: For every probability measure $\mu$ on $V_{k}$, there is $1 \leq i \leq n$ with $\left|\int f_{i}(x) d \mu(x)-\int f_{i}\left(g_{i} x\right) d \mu(x)\right|>\varepsilon$.

Indeed, assuming the contrary to 1.8 yields, using a standard weak limit argument, a $G_{k}$-invariant, finitely additive probability measure on $V_{k}$, thereby contradicting 1.6. (For a precise argument see Definition 4.1 and use 5.6 below.)

Both proofs of our main results, Theorems 1.1 and 1.6, are based on a fundamental and important feature of algebraic actions, namely, their smoothness (called also "tameness"- see Def. 2.1 below). Our strategy in proving 1.1 is to reduce the analysis, using this smoothness, to the one parameter subgroups $G_{a}, G_{m}$, which form the "bricks" from which the proof is built. In the proof of 1.6, $S L_{2}$ subgroups play a similar role. For $\sigma$-additive measures, our approach yields a particulary simple proof of Borel's density theorem for semisimple groups (see the remark proceeding 3.9 ), and enables one to treat uniformly the different groups also over positive characteristic (which apparently has not been studied yet in general, in that context). We remark that for our purposes, solvable groups over positive characteristic can 
behave quite differently than the ones in characteristic zero. (We mention here [40], where Borel's density theorem for solvable groups is essential.) Using this approach to the study of $\sigma$-additive invariant measures, we shall also be able to strengthen and present other known results (e.g. of Dani [10], Tits [37], Wang [39] and BorelSerre [7]) from a more general point of view, while for finitely additive measures, which are in general quite difficult to handle, our method yields a complete analysis of these measures in the framework of algebraic actions. We remark that as the paper is intended also for non-experts in the general theory of algebraic groups, we shall assume only basic familiarity with this theory. We should also note here that after this paper had been completed, [1] was called to our attention, in which the smoothness of algebraic actions is used in a similar flavour to ours, in a proof of Borel's density theorem over $\mathbb{R}$ (the use of Poincaré recurrence theorem is special to $\mathbb{R}$, see also Gromov's proof there).

Theorem 1.6 has several spectral consequences. Before elaborating on the main one, which is the subject of Section 5, observe that our first application of Theorem 1.6, Corollary 1.7, could have also been stated in representation theoretic terms: By a result of Eymard [12], the space $G_{k} / \Gamma$ supports a probability $G_{k}$-invariant, finitely additive measure, iff the $G_{k^{-}}$(natural) unitary representation on $L^{2}\left(G_{k} / \Gamma\right.$ ) weakly contains (or, in other words, is not bounded away from) the trivial representation-see Theorem 4.18 below. Therefore, Corollary 1.7 can be restated as follows: Under the same condition on $G$ as in 1.7, if $\Gamma<G_{k}$ is not Zariski dense, then the $G_{k}$-representation on $L^{2}\left(G_{k} / \Gamma\right)$ is bounded away from the trivial representation. It turns out that the proof of Theorem 1.6 is flexible enough to deal also with countable (disjoint) unions of algebraic varieties. Consequently, we will show in 4.20 that in the latter reformulation of Corollary 1.7, not only the one $G_{k}$-representation on $L^{2}\left(G_{k} / \Gamma\right)$, but actually all the representations of this type together, when $\Gamma$ varies over all the non-Zariski dense subgroups of $G_{k}$, are bounded away from the trivial representation.

In Section 5 we present the main representation theoretic application to our study of finitely additive invariant measures. We show how questions about existence and uniqueness of such measures are both natural and fruitful in the investigation of Kazhdan's property $(T)$ and variants of it (see 5.5, 5.7 below). For instance, we use Theorem 1.6 to show:

1.9 Theorem. Let $G$ be a $k$-group and $N$ its $k$-disamenable radical as defined in 1.5 above. Then $G_{k}$ has property $(T)$ iff $G_{k} / R a d G_{k}$ has property $(T)$, and $G_{k} / N_{k}$ is compact.

A completely different approach to this characterization of algebraic groups with property $(T)$ was suggested by Wang [38]. Here we strengthen Wang's results and show that in general (but not always!) actually a stronger property holds for $k$ groups with property $(T)$, namely, there exists a finite set $F \subseteq G$ and $\varepsilon>0$ such that if $\pi$ is a unitary representation containing an $(F, \varepsilon)$ invariant vector (Def. 5.1 ), then $\pi$ must contain an invariant vector. (The replacement of compact by finite is, roughly, due to the finite intersection property in the weak-* compact space of finitely additive probability measures.) For example, we show in 5.13 that a Kazhdan $(k$-)group has this stronger property $(T)$ if it has no non-trivial $k$-compact quotients. We also study general connected Lie groups in that context, and discuss compact groups, which are of special interest. 
Our investigation of the uniqueness and existence of finitely additive invariant measures should also be compared with the results in [13], [14]. It is shown there how these questions have purely ergodic theoretic implications. Thus, the analysis of invariant measures for algebraic actions leads to generalizations of Borel's fixed point and density theorems (and the latter's extension by Stuck), as well as representation and ergodic theoretic consequences. In this paper we shall not deal with the measure theoretic applications, but merely indicate some connections between the above different themes, which arise via our study of invariant measures.

\section{ACKNOWLEDGMENTS}

I would like to thank deeply and warmly Hillel Furstenberg for many illuminating discussions and valuable suggestions, regarding various aspects connected to the paper. Special thanks to A. Nevo for helpful remarks on the manuscript. Thanks also to G. Prasad and A. Rapinchuk for useful information on algebraic groups over positive characteristic, and to D. Witte and R. Zimmer for some useful discussions.

\section{Preliminaries}

We briefly review some notions and results which are of constant use in the sequel.

2.1 Definition. I: A Borel space is called countably separated if there is a sequence of Borel sets which separate points.

II: Let $S$ be a countably separated Borel $G$ space ( $G$-l.c. group). The action of $G$ on $S$ is called smooth (or tame) if the quotient Borel structure on $G \backslash S$ is countably separated.

One of the useful features of smooth actions is:

2.2 Lemma. Suppose $G$ is a l.c. group, $H<G$, and that $G$ acts smoothly on $S$. Then every $H$-quasi-invariant ergodic $\sigma$-additive measure on $S$ is supported on a G-orbit.

For a proof see [41, 2.1.10]. The proof given there for the case $H=G$ is valid in the general case.

If $G$ and $V$ are algebraic group/variety defined over $k$, we denote by $G_{k}, V_{k}$ their $k$-points. The following result which follows from a theorem of Borel-Serre [6] (when char $k=0$ ), and Bernstein-Zelevinski $[2,6.15]$ (for any local field), together with the observation made in [41, 2.1.12], will play an important role in the sequel:

2.3 Theorem. If a $k$-group $G$ acts $k$-regularly on a $k$-variety $V$, then the action of $G_{k}$ on $V_{k}$ is smooth, where $V_{k}$ is endowed with the natural Borel structure coming from the locally compact topology of $k$.

We remark that a number of natural and important group actions have the smoothness property- see [41, Chapter 3].

2.4 Definition. If $G$ is a $k$-group we call $G k$-compact ( $k$-amenable) if $G_{k}$ is compact (amenable). If $H \triangleleft G$ we call $H k$-cocompact (k-coamenable) if $G_{k} / H_{k}$ is compact (amenable). 
2.5. If $H<G$ (k-groups) then there is a natural $G_{k}$-embedding $G_{k} / H_{k} \hookrightarrow(G / H)_{k}$ which is not necessarily surjective. If char $k=0$ there are only finitely many $G_{k^{-}}$ orbits in $(G / H)_{k}$ [6]. In particular, if $H \triangleleft G$, then $G_{k} / H_{k}$ has finite index in $(G / H)_{k}$, so if $H$ is $k$-cocompact ( $k$-coamenable) then $G / H$ is $k$-compact ( $k$-amenable). When char $k>0$ this is no longer true in general. We remark that it will develop (3.14) that if $G$ has any proper normal subgroup which is $k$-cocompact, then it has a (perhaps different) proper normal $k$-subgroup with $k$-compact quotient.

2.6. When dealing with local fields of arbitrary characteristic one has to be careful with separability problems. If char $k>0$ the notions " $k$-closed" and "defined over $k$ " do not coincide, so, for example, if $G$ is a $k$-group acting $k$-regularly on a $k$ variety $V$, then Fix $G$ - the fixed points of $G$, is $k$-closed but not necessarily defined over $k$ (cf. [19]). As it will be important for us to have our objects defined over $k$, we will use the following: Suppose $V$ is a variety with a $k$-structure, and $W \subseteq V$ is any subset. We then define

$$
W_{k}=W \cap V_{k} .
$$

Obviously, if $W$ is a $k$-subvariety this agrees with the usual notation. Since $W_{k} \subseteq$ $V_{k}$, it follows from [4, p. 57] that its Zariski closure $\overline{W_{k}}$ is always defined over $k$. As the objects we are really interested in, are the $k$-points of sets (on which, for example, measures are defined), we shall often replace $W$ by $\overline{W_{k}}$, thereby getting a $k$-subvariety whose $k$-points contain the " $k$-points" of $W$.

We close this section with a basic result which will become very useful in the sequel:

2.7 Lemma. Suppose that $G$ is a connected $k$-group which acts k-regularly on a $k$-variety $V$, and $\mu$ is any finitely additive probability measure on $V_{k}$. Then there exists a maximal connected $k$-subgroup $H<G$ with the property that $\mu(\text { Fix } H)_{k}=1$. (Here FixH $=\{v \in V \mid h v=v \forall h \in H\}$ and $(\text { FixH })_{k}$ is as defined in 2.6). $H$ is called the fixing subgroup of $\mu$. Furthermore, if $\mu$ is $G_{k}$-quasiinvariant (i.e. $\mu(A)=0 \Rightarrow \mu(g A)=0 \quad \forall g \in G_{k}$ ), then $H \triangleleft G$.

Proof. Let $\left\{H_{\alpha}\right\}$ be the family of all such $k$-subgroups ( $\left\{H_{\alpha}\right\}$ obviously contains $\{e\})$. Define $V_{\alpha}=$ FixH $_{\alpha}$ and $W=\bigcap V_{\alpha}$. By the descending chain condition for closed subvarieties $W=V_{\alpha_{i}} \cap \cdots \cap V_{\alpha_{n}}$ so $W_{k}=\left(V_{\alpha_{1}}\right)_{k} \cap \cdots \cap\left(V_{\alpha_{n}}\right)_{k}$, and since $\mu\left(V_{\alpha_{i}}\right)_{k}=1$ for all $1 \leq i \leq n$ we have $\mu\left(W_{k}\right)=1$. Let $H^{k}=\left\{g \in G_{k} \mid g w=w \forall w \in\right.$ $W\}$ and $H=\overline{H^{k}}$. Then by $2.5 H$ is defined over $k$ and since $\left(H_{\alpha}\right)_{k} \subseteq H^{k}$ for all $\alpha, H_{\alpha} \subseteq H$ (recall that if $k$ is any local field then $G_{k}$ is Zariski dense in $G$, for $G$ connected and defined over $k$ - see e.g. [26, I.2.5.3(ii)]). As $\mu(F i x H)_{k} \geq \mu\left(W_{k}\right)=1$, the connected component of $H$ is clearly maximal with the above property, and the first assertion follows.

Next, Fix $\left(g \mathrm{Hg}^{-1}\right)=g($ Fix $H)=g W$ and if $g \in G_{k}$ we may consider the equality for the $k$-points (in the sense of 2.6) as well. If $\mu$ is $G_{k}$-quasi-invariant, the measure of (the $k$-points of) the right hand side, and therefore that of the left hand side as well, is 1 , so $g H g^{-1} \in\left\{H_{\alpha}\right\}$. By the maximality of $H, g H g^{-1} \subseteq H$, hence $g H g^{-1}=H$ for all $g \in G_{k}$. Since $G_{k}$ is Zariski dense in $G$, indeed $H \triangleleft G$.

\section{3. $\sigma$-Additive invariant measures - Proof of Theorem 1.1}

In this section $k$ has arbitrary characteristic. Recall (2.6) that if $V$ is a $k$-variety and $W \subseteq V$ is any subset, we define $W_{k}=W \cap V_{k}$, and $\overline{W_{k}}$ is always defined over 
$k$. The strategy in proving Theorem 1.1 is to consider first the "in particular" part stated at the end of the theorem. We then prove and use the structure theorem stated in 1.4 to deduce the general case.

In this section we shall use the abbreviated notation $(G, V, \mu)$ to denote the following setup: $G$ is a $k$-group which acts $k$-regularly on a $k$-variety $V$, and $\mu$ is a $\sigma$-additive probability measure on $V_{k}$, invariant under the action of $G_{k}$. For brevity, we shall say that $(G, V, \mu)$ is a $G$-system.

3.1 Definition. We say that $G$ has the Borel property (abbreviated notation: B.pr.) if whenever $(G, V, \mu)$ is a $G$-system, then $\mu(\text { Fix } G)_{k}=1$.

As we shall easily observe, the Borel density theorem is equivalent to saying that if $G_{k}$ is semisimple with no compact factors then $G$ has the B.pr.

3.2 Lemma. Let $H, G$ be connected k-groups. If $H \triangleleft G$, and $G$ has the B.pr., so does $G / H$. If $H$ and $G / H$ have the B.pr., then so does $G$.

Proof. The first statement is obvious. To prove the second suppose $(G, V, \mu)$ is a $G$-system, let $W^{k}=(\text { FixH })_{k}=F i x\left(H_{k}\right) \cap V_{k}$, and $W=\overline{W^{k}}$ (defined over $k$ by 2.6). $W^{k}$ is the fixed point set for the action of $H_{k}$ on $V_{k}$ and since $H_{k} \triangleleft G_{k}, W^{k}$ is $G_{k}$-invariant and $W$ is $G$-invariant. Furthermore, the action of $G$ on $W$ factors through $G / H$ and as $H$ has the B.pr., $\mu\left(W_{k}\right)=1$. Now apply the B.pr. of $G / H$ for its action on $W$, and the proof is complete.

3.3 Theorem. The following conditions on a connected $k$-group $G$ are equivalent: 1. G has the B.pr.

2. If $H<G$ is a k-subgroup and on $G_{k} / H_{k}$ there is a $G_{k}$-invariant probability measure, then $G=H$.

3. If $(G, V, \mu)$ is a $G$-system, then there exists a $G$-fixed point in $V$.

Proof. $1 \Rightarrow 2$ : The proof is identical to that in 1.2 .

$2 \Rightarrow 3$ : Existence of an invariant measure implies that there is also an ergodic one. By 2.3 the action of $G_{k}$ on $V_{k}$ is smooth and applying 2.2 shows that $\mu$ (which is now assumed to be ergodic) is supported on an orbit $G_{k} v_{0}$. Let $H^{k}=\{g \in$ $\left.G_{k} \mid g v_{0}=v_{0}\right\}$ and $H=\overline{H^{k}}$. Then $H v_{0}=v_{0}$, so $H_{k}=H^{k}$ and $G_{k} v_{0} \sim G_{k} / H_{k}$ as $G_{k}$-Borel spaces (see [41, A.4], actually they are homeomorphic). Thus $\mu$ may be regarded as a $G_{k}$-invariant measure on $G_{k} / H_{k}$. By the assumption $G=H$ so $v_{0}$ is fixed by $G$.

$3 \Rightarrow 1$ : Suppose $(G, V, \mu)$ is a $G$-system, and define $W=\overline{(F i x G)}_{k} . W$, and therefore $V-W$, are $G$-invariant $k$-subvarieties ( $W$ closed, $V-W$ open). Since there is no $G$-fixed point in $V-W$ it follows from 3 that $\mu\left(V_{k}-W_{k}\right)=0$, so $\mu\left(W_{k}\right)=1$ and this completes the proof.

3.4 Corollary. The additive and multiplicative groups $G_{a}, G_{m}$ have the B.pr.

Proof. Follows from 2 in 3.3, as any proper algebraic subgroup of them is finite.

The following notion will play an essential role in the sequel:

3.5 Definition. A $k$-group $G$ is said to be $k$-discompact if whenever $\varphi: G \rightarrow H$ is a surjective $k$-homomorphism and $H$ is $k$-compact then $\varphi$ is trivial. This is equivalent to the seemingly more general property where the same holds without assuming that $\varphi$ is surjective (using the fact that $\varphi(G)$ is always a $k$-subgroup of $H)$. 
Notice that $k$-discompact groups are in particular connected since the connected component is a normal $k$-subgroup of finite index. Before stating the next result we recall that a solvable $k$-group is called $\mathbf{k}$-splitting if it has a composition series of $k$-subgroups in which each successive quotient is $k$-isomorphic to either $G_{a}$ or $G_{m}$, and it is called k-anisotropic if it has no $k$-subgroup which is $k$-splitting.

3.6 Theorem. Let $G$ be a connected solvable k-group and consider the following properties:

1. $G$ is k-discompact.

2. $G$ is k-splitting.

3. G has the B.pr.

4. $G$ satisfies the fixed point property in condition 2 of 1.3.

5. $G$ is k-triangulizable.

Then (1)-(4) are equivalent and they imply (5). If char $k=0$ all the properties are equivalent.

Proof. We start by proving $1 \Rightarrow 5$ and notice the following: A careful examination of Furstenberg's Lemma [15, Lemma 3] shows that although it is stated and used for semisimple groups, actually a more general statement is proven there: Let $V$ be a finite dimensional vector space over $k$ and $G \subset G L(V)$ any subgroup (not necessarily algebraic). Suppose that: (1) Every subspace $W \subseteq V$ which is invariant under a subgroup of finite index in $G$ is actually invariant under $G$, and (2): If $W \subseteq V$ is a non-zero $G$-invariant subspace and the image of $G$ in $P G L(W)$ is precompact then this image is in fact trivial. Then every probability measure on the projective space $P(V)$, which is invariant under the projective action of $G$, is supported on $G$-fixed points in $P(V)$.

Proof of $1 \Rightarrow 5$. Embed $G \subseteq G L_{n}=G L(V)$ as a $k$-subgroup. Since $G_{k}$ is solvable, it is amenable, and has an invariant probability measure $\mu$ for its action on $P\left(V_{k}\right)$. $G$ is $k$-discompact therefore the two conditions of Furstenberg's Lemma as stated above are satisfied for the action of $G_{k}$ on $V_{k}$. Since $\mu$ is $G_{k}$-invariant we obtain an invariant $k$-line $L$. Now take $V_{1}=V / L$ and continue by induction to get an invariant $k$-flag.

$1 \Rightarrow 2$ : Using $1 \Rightarrow 5$ we may assume that $G$ is a $k$-subgroup of the triangular group, so its maximal torus is obviously $k$-splitting, and it suffices to show that so is its unipotent radical, $G_{u}$ (which is always defined over $k$ ). Let $H \triangleleft G_{u}$ be the maximal $k$-splitting subgroup ([37, III 4.2]). By the maximality of $H$, the unipotent radical of $G / H$ is $k$-anisotropic and it follows ([37, III 5.3.1]) that the conjugation action of any maximal torus (which is $k$-splitting by the above) on it, is trivial. Such a torus is therefore central and by dividing it out we get a $k$-image of $G$ which is $k$-anisotropic and therefore, by a result of Wang [39], $k$-compact. Since $G$ is $k$-discompact this image is trivial, so $H=G_{u}$ as required.

$2 \Rightarrow 3$ : Consider a composition series: $\{e\}=G_{n} \triangleleft \ldots \triangleleft G_{0}=G$, in which for every $i: G_{i} / G_{i+1}$ is $k$-isomorphic to either $G_{a}$ or $G_{m}$. By 3.4 both $G_{a}$ and $G_{m}$ have the B.pr. and using 3.2 we can work our way up from $\{e\}$ to $G$, so $G$ has the B.pr. as well.

$3 \Rightarrow 4$ : This is what is actually shown in the proof of $1.3,1 \Rightarrow 2$.

$4 \Rightarrow 1$ : See $1.3,2 \Rightarrow 1$.

If char $k=0$ every $k$-triangulizable group splits over $k$ (see e.g. $[4,15.4]$ ), so all the five properties are equivalent. The proof of the theorem is now complete. 
We remark that in case $\operatorname{char} k=0$ a much simpler proof of 3.6 can be given. This is also the case for the structure theorem formulated in 1.4, which we presently prove:

3.7 Proof of 1.4. Let $\left\{N_{\alpha}\right\}$ be the family of all the $k$-discompact subgroups of $G$, and $N$ the smallest closed subgroup of $G$ containing all of them. $N$ is defined over $k$ ([4, p. 106]- note that the $N_{\alpha}$ 's are connected) and if $\varphi: N \rightarrow H$ with $H$ $k$-compact, $\operatorname{ker} \varphi$ contains each of the $N_{\alpha}$ 's, so by the minimality of $N, \operatorname{ker} \varphi=N$. It follows that $N$ is $k$-discompact and is obviously maximal. The second statement of 1.4 follows readily from the maximality of $N$ and we now prove the last one.

If $G$ is $k$-discompact then $N=G$ and we are done. Otherwise, let $\varphi_{1}: G \rightarrow H^{1}$ be such that $H_{k}^{1}$ is compact, and denote $G^{1}=\overline{\operatorname{ker} \varphi_{1} \cap G_{k}}$. The group $G^{1}$ is defined over $k$ and $G_{k} / G_{k}^{1} \cong \varphi\left(G_{k}\right)$ is compact, being (open and) closed subgroup of $H_{k}^{1}$. Now, if $G^{1}$ is not $k$-discompact, there exists $\varphi_{2}: G^{1} \rightarrow H^{2}$ with $H_{k}^{2}$ compact and we may continue this process by defining $G^{i}=\overline{\operatorname{ker} \varphi_{i} \cap G_{k}}, \varphi_{i+1}: G^{i} \rightarrow H^{i+1}\left(H_{k}^{i}\right.$ compact). Since $N$ is $k$-discompact, necessarily $N<G^{i}$ for all $i$. As above, all $G_{k}^{i} / G_{k}^{i+1}$ are compact. By d.c.c. the chain ... $G^{2} \triangleleft G^{1} \triangleleft G$ terminates, say in $G^{n}$. As the sequence terminates in $G^{n}$ this group is $k$-discompact, so by the maximality of $N$ as a $k$-discompact subgroup, $N=G^{n}$. From the compactness of each $G_{k}^{i} / G_{k}^{i+1}$ it follows that $G_{k} / N_{k}$ is compact, and if char $k=0$ this suffices since this group has finite index in $(G / N)_{k}(2.5)$.

To complete the proof for a general $k$ we prove:

3.8 Lemma. If a connected $k$-group $G$ has no non-trivial solvable $k$-subgroup which is k-discompact (equivalently, by 3.6: k-splitting, or has the B.pr.), then $G$ is $k$ compact.

Proof of 3.8. First assume $G$ is solvable. Then the assumption amounts to saying that the $k$-discompact radical of $G$ is trivial so by the argument above $G$ is $k$ compact. For a general $k$-group $G$ denote $R=\operatorname{Rad} G$ and we show that both $R$ and $G / R$ are $k$-compact (which clearly implies that so is $G$ ). The assumption on $G$ passes to $R$, so by the solvable case $R$ is $k$-compact. If the semisimple group $G / R$ is not $k$-compact, then $k$-rank $G>0$ (see e.g. [29]), so it contains a $k$-isotropic torus and therefore so does $G$, contradicting the assumption. The proof of 3.8 is complete.

We now finish the proof of Proposition 1.4: Let $N$ be the $k$-discompact radical of $G$. By the maximality of $N, G / N$ has no $k$-discompact subgroups. Applying 3.8 completes the proof.

As a consequence of Theorems 1.4 and 3.6, we can describe the structure of solvable $k$-groups as follows: the $k$-discompact radical is the maximal $k$-splitting subgroup, and the quotient of the group by this maximal $k$-splitting subgroup is $k$-compact. In particular, $G$ is $k$-discompact iff it is $k$-splitting and it is $k$-compact iff it is $k$-anisotropic (the latter is Wang's result [39], which was used in the proof. Compare also with the general structure theorem in [37, III]).

We are now in position to prove the "in particular" part of 1.1, in the following formulation:

3.9 Theorem. If $G$ is k-discompact then $G$ has the B.pr.

Proof. First notice that the converse is obvious, again, by considering the (multiplication invariant) Haar measure on any $k$-compact image. Suppose now that $G$ is 
$k$-discompact and let $(G, V, \mu)$ be a $G$-system. Let $H \triangleleft G$ be the fixing $k$-subgroup of $\mu$ (2.7). By the maximality of $H, G / H$ does not contain any non-trivial $k$-subgroup which has the B.pr., and it follows from 3.8 that $G / H$ is $k$-compact. Since $G$ is $k$-discompact necessarily $H=G$, as required.

Remark. Most of the difficulty in the proof of 3.9 is due to the solvable case. For the Borel density theorem we need to consider only semisimple groups, and the argument for them is considerably simpler. By 3.2 it is enough to prove the theorem when $G$ is almost $k$-simple and not $k$-compact: Suppose $(G, V, \mu)$ is a $G$-system and let $H \triangleleft G$ be the fixing subgroup of $\mu$. Taking any non-trivial $k$-isotropic torus $T \subseteq G$ ( $k$-rank $G>0$ ), we see that by $3.4 T \subseteq H$. It follows that $\operatorname{dim} H>0$ and by almost $k$-simplicity $H=G$, as required.

Let us now complete the proof of the general theorem.

3.10 Proof of 1.1. Let $N \triangleleft G$ be the $k$-discompact radical and $H=G / N$. By $1.4 H_{k}$ is compact, and by $3.9 N$ has the B.pr. If $(G, V, \mu)$ is a $G$-system, let $W=\overline{(F i x N)_{k}}$. Then $W$ is defined over $k, \mu\left(W_{k}\right)=1$ and everything follows immediately.

Notice that in light of 1.1 the $k$-discompact radical of $G$ may be characterized either as the largest $k$-discompact subgroup of $G$, or as the smallest $k$-subgroup $H$ such that on $G_{k} / H_{k}$ there is a $G_{k}$-invariant probability measure (more generally, it is contained in the Zariski closure of any "finite co-volume" subgroup of $G_{k}$ ). In fact it is the unique $k$-subgroup which has both the $k$-discompactness and the "finite co-volume" properties.

Let us concentrate now on the special case $k=\mathbb{R}$. The stabilizer in $P G L_{n}(\mathbb{R})$ of any probability measure on the projective space $\mathbb{P}^{n-1}(\mathbb{R})$ is (the $\mathbb{R}$-points of) an algebraic group ([41, 3.2.4)]. If $H$ is an $\mathbb{R}$-group, then using Chevalley's theorem, the action of $G$ on $G / H$ can always be $\mathbb{R}$-embedded in such an action, and thus stabilizers in $G_{\mathbb{R}}$ of measures on $G_{\mathbb{R}} / H_{\mathbb{R}}$ are algebraic. We now extend this observation, replacing the action on $G / H$ by any $\mathbb{R}$-action on a $\mathbb{R}$-variety $V$ : Let $\mu$ be any probability measure on $V_{\mathbb{R}}$, denote by $\left(G_{\mathbb{R}}\right)_{\mu}$ the stabilizer of $\mu$, and by $M$ (the $\mathbb{R}$-points of) its Zariski closure in $G$. We want to show that $M$ preserves $\mu$. Indeed, write an ergodic decomposition of $\mu: \mu=\int \mu_{s} d v(s)$. For $v$-a.e. $s, \mu_{s}$ is $\left(G_{\mathbb{R}}\right)_{\mu}$-invariant and since it is ergodic it follows from $2.2,2.3$ that every such $\mu_{s}$ is supported on a $G_{\mathbb{R}}$-orbit. By the argument above, $\mu_{s}$ is preserved by $M$, and as this is the case for $v$-a.e. $s, \mu$ is $M$-invariant. Thus, applying this remark together with 1.1 we deduce the following result (proved by Dani [10], when $V=G / H$ ):

3.11 Theorem. If $G$ is an $\mathbb{R}$-group acting $\mathbb{R}$-regularly on a variety $V$, and $\mu$ is any probability measure on $V_{\mathbb{R}}$, then the stabilizer of $\mu$ in $G_{\mathbb{R}}$ is algebraic and has a normal cocompact subgroup which fixes every point in the support of $\mu$.

We end this section with some results which are both useful for the next section and may be of independent interest.

3.12 Proposition. Let $G$ be a k-group and $R=\operatorname{Rad} G$. Then $G$ is $k$-discompact iff both $R$ and $G / R$ are $k$-discompact.

Proof. Obviously, the only non-trivial statement is that the $k$-discompactness of $G$ implies that of $R$. Let $R_{1}$ be the $k$-discompact radical of $R$, so it suffices to prove that $R_{1}=R$. By $1.4 R_{1} \triangleleft G$. Letting $H=G / R_{1}$ we deduce that $H$ is $k$-discompact 
and $(\operatorname{Rad} H)_{k} \cong\left(R / R_{1}\right)_{k}$ is compact (by 1.4). $H$ acts by conjugation on its radical, so $H_{k}$ preserves the (finite) Haar measure of $(\operatorname{RadH})_{k}$. By $3.9 H$ has the B.pr. so the Haar measure is supported on $H$-fixed points. It follows that $H$ acts trivially on its radical, which is therefore central. Thus, $\operatorname{Rad} H$ is isomorphic to the quotient of $H$ by its maximal semisimple $k$-subgroup (which exists in this case, also when char $k>0$ ), so it is both $k$-discompact and $k$-compact, hence trivial, implying that $R_{1}=R$.

The following straightforward consequence of 3.12 and 3.6 will be used in the next section:

3.13 Corollary. If $G$ is $k$-discompact then RadG can be triangulized over $k$.

Finally, we prove the remark alluded to at the end of 2.5:

3.14 Proposition. If $G$ has a normal $k$-cocompact proper $k$-subgroup $H$, then $G$ has a normal proper $k$-subgroup $N$ such that $G / N$ is $k$-compact.

Proof. If $G$ does not have such a subgroup $N$, by $1.4 G$ is $k$-discompact so it has the B.pr. (3.9). $G_{k} / H_{k}$ supports a $G_{k}$-invariant (Haar) measure and via the natural $G_{k}$-embedding: $G_{k} / H_{k} \hookrightarrow(G / H)_{k}$ we obtain a $G_{k}$-invariant measure for its action on $V_{k}$, where $V=G / H$. Since $G$ has the B.pr. there is a $G$-fixed point in $G / H$ so $H=G$, a contradiction.

\section{Finitely additive invariant measures - Proof of Theorem 1.6}

Hereafter, to avoid some technical difficulties, we assume that $\operatorname{char} k=0$. Our strategy in proving Theorem 1.6 (stated in the introduction) is to reduce it to the case $G=S L_{2}$, whose consideration occupies most of the proof. We first introduce the commonly used notation:

4.1 Definition. A charge $\mu$ on a Borel space $X$ is a finitely additive probability measure (i.e. $\mu(X)=1$ ), defined on all the Borel subsets.

Modifying the notation used in section $3,(G, V, \mu)$ will stand for an abbreviated notation of the setup where a $k$-group $G$ acts $k$-regularly on a $k$-variety $V$, and $\mu$ is a charge on $V_{k}$ (with its natural Borel structure), which is invariant under the action of $G_{k}$. For brevity, we now call $(G, V, \mu)$ an additive $G$-system. Analogous to 3.1 and 3.5 we define:

4.2 Definition. We say that a $k$-group $G$ has the strong Borel property (abbreviated notation: st.B.pr.) if whenever $(G, V, \mu)$ is an additive $G$-system, then $\mu(\text { FixG })_{k}=1$.

4.3 Definition. A $k$-group $G$ is said to be k-disamenable if whenever $\varphi: G \rightarrow H$ is a surjective $k$-homomorphism with $H_{k}$ amenable, then $\varphi$ is trivial. As in 3.5, an equivalent property is obtained by considering $\varphi: G \rightarrow H$ which are not necessarily surjective. (For definition of amenability, the last statement of 4.18 below may be used).

Example. If $G$ is semisimple and $G_{k}$ has no compact factors then $G$ is obviously $k$-disamenable. The semi-direct product $S L_{n}(K) \ltimes K^{n}$ with the natural $k$-structure, is another example of such a $k$-group.

By exactly the same arguments as in the first part, before 3.8, of the proof of 1.4, we have the following analogue (replacing "compact" by "amenable"): 
4.4 Proposition. Every $k$-group $G$ has a maximal $k$-disamenable subgroup $N$, i.e. $N$ is $k$-disamenable and contains every $k$-disamenable subgroup of $G . N$ is characteristic for $k$-automorphisms of $G$, in particular $N \triangleleft G$. Moreover, $(G / N)_{k}$ is amenable. $N$ is called the $k$-disamenable radical of $G$.

Using the following observation we shall identify the $k$-disamenable radical.

4.5 Lemma. If $N \triangleleft G, N$ is $k$-disamenable and $(G / N)_{k}$ is amenable, then $N$ is the $k$-disamenable radical of $G$.

Proof. If $H$ is any $k$-disamenable subgroup then the image of it in the projection $G \rightarrow G / N$ must be trivial, so $H \subseteq N$.

4.6 Proposition. Let $g_{k}=h_{k} \oplus r_{k}$ be a Levi-Malcev decomposition of $\mathfrak{g}_{k}=$ $\operatorname{Lie}\left(G_{k}\right)$ and write $\mathfrak{h}_{k}=\mathfrak{h}_{k}^{c} \oplus \mathfrak{h}_{k}^{n}$ a sum of the "compact" and "non-compact" ideals of the semisimple part $-\mathfrak{h}_{k}$. Let $H^{c}, H^{n}$ be the semisimple $k$-subgroups of $G$ corresponding to $\mathfrak{h}^{c}=\mathfrak{h}_{k}^{c} \otimes K, \mathfrak{h}^{n}=\mathfrak{h}_{k}^{n} \otimes K$ and let $N$ be the smallest normal $k$-subgroup of $G$ containing $H^{n}$ (notice that $H_{k}^{n}$ is a maximal semisimple subgroup of $G_{k}$ with no compact factors). Then $N$ is the $k$-disamenable radical of $G$.

In particular, $G$ is $k$-disamenable iff both $(G / R a d G)_{k}$ has no compact factors and there is no proper normal $k$-subgroup of $G$ containing a maximal semisimple $k$-subgroup.

Proof. We show that $N$ satisfies the criterion of 4.5. Clearly $N$ is $k$-disamenable since $H^{n}$ and all its conjugates are. To check the second condition notice that the $k$-points of the $k$-group which corresponds to $\mathfrak{h}^{c} \oplus r$ is amenable (compact extension of solvable) and that it projects onto $(G / N)$ 。 (connected component).

Remark. Proposition 3.12 shows that the $k$-discompactness of a group depends only on its semisimple and radical parts and not on the way the former acts on the latter. Proposition 4.6 shows that the nature of this action determines if the group is $k$-disamenable.

As in Section 3 the "in particular" part of 1.6 amounts to saying that if $G$ is $k$-disamenable then $G$ has the st.B.pr. (the converse is obvious as in the $\sigma$-additive case, once we recall that amenable groups are characterized by the existence of an invariant charge for their self action). The same proof as in 3.10 shows that using 4.4, the general statement of Theorem 1.6 follows from the "in particular" one. We have therefore established the following:

4.7 Lemma. To prove 1.6 it suffices to see that $k$-disamenable groups have the st.B.pr.

We reduce further:

4.8 Lemma. To prove 1.6 it suffices to show that if $G$ is connected semisimple and almost $k$-simple, such that $G$ is not $k$-compact, then $G$ has the st.B.pr.

Proof. By 4.7 it is enough to show that $k$-disamenable groups have the st.B.pr. Let $G$ be such a group, suppose $(G, V, \mu)$ is an additive $G$-system, and let $H \triangleleft G$ be the fixing subgroup of $\mu$, as guaranteed in 2.7. By the assumption of $4.8 \mathrm{H}$ contains all the almost $k$-simple non- $k$-compact subgroups of $G$. However, by 4.6 the $k$-disamenability of $G$ implies that there is no proper normal $k$-subgroup of $G$ with this property, and therefore $H=G$ as required.

The final reduction is: 
4.9 Lemma. To prove 1.6 it suffices to show that $S L_{2}$ has the st.B.pr.

Proof. By 4.8 we merely need to show that if $G$ is connected almost $k$-simple and not $k$-compact then $G$ has the st.B.pr. Suppose $(G, V, \mu)$ is an additive $G$-system, and again let $H \triangleleft G$ be the fixing subgroup of $\mu$. Since $G$ is not $k$-compact, $k$-rank $G>0$ and in $\mathfrak{g}_{k}=$ Lie $G_{k}$ there is $X \neq 0$ which is $a d_{\mathfrak{g}}$ nilpotent. By the JacobsonMorozov theorem (see e.g. $[18,7.4]$ ), $X$ is contained in a subalgebra $k$-isomorphic to $s l_{2}$. As every algebraic group having $s l_{2}$ Lie algebra is covered by $S L_{2}$, we conclude from the assumption that $G$ contains a (non-trivial) $k$-subgroup which has the st.B.pr. Therefore $\operatorname{dim} H>0$ and since $G$ is almost $k$-simple, $H=G$.

To discuss $S L_{2}$ we first mention the following lemma, which is a special case of a result described in 4.18 (see also Definition 4.17).

4.10 Lemma. Suppose $G$ is any locally compact topological group, $H<G$ a closed subgroup and that there is a $G$-invariant charge on $G / H$. If $H$ is amenable, then so is $G$.

We now notice that in Theorem 3.3 , the proof of the implication $3 \Rightarrow 1$ carries through without any change to the case of charges and the st.B.pr. Therefore to prove that $S L_{2}$ has the st.B.pr. it suffices to show that if $\left(S L_{2}, V, \mu\right)$ is an additive $S L_{2}$-system then $S L_{2}$ has a fixed point in $V$. However, the proof of the equivalence of these two conditions with condition 2 completely fails when $\mu$ is not $\sigma$-additive, and this causes the difficulty in the proof of 1.6. Since the $k$-points of every proper $k$-subgroup of $S L_{2}$ is solvable (and hence amenable), while $S L_{2}(k)$ is not, condition 2 (for charges) in 3.3 is completely satisfied for $G=S L_{2}$, in view of 4.10. A posteriori, the equivalence of these three conditions (for charges) will be established, but only as a corollary to 1.6 (and not as a tool in its proof).

Two features of the algebraic group $S L_{2}$ make it convenient to work with. The first is that all its proper algebraic subgroups are solvable and the second is that one can actually list all its $k$-subgroups:

4.11 Lemma. Let $H<S L_{2}$ be a (not necessarily connected) proper $k$-subgroup. Then $\mathrm{H}_{k}$ is conjugated in $\mathrm{SL}_{2}(k)$ to one of the following subgroups:

1. A countable family of finite groups, all of them contained in a given maximal compact (not necessarily algebraic) subgroup $C<S L_{2}(k)$.

2. A countable family of groups of the form $\left(\begin{array}{cc}\lambda & \alpha \\ 0 & \lambda^{-1}\end{array}\right)$ where $\alpha \in k$, and $\lambda \in k^{*}$ is taken from a finite group of roots of unity.

3. $\left\{\left(\begin{array}{cc}a & 0 \\ 0 & a^{-1}\end{array}\right) \mid a \in k^{*}\right\}$.

4. $\left\{\left(\begin{array}{cc}a & 0 \\ 0 & a^{-1}\end{array}\right)\left(\begin{array}{cc}0 & b \\ -b^{-1} & 0\end{array}\right) \mid a, b \in k^{*}\right\}$.

5. $\left\{\left(\begin{array}{cc}a & b \\ 0 & a^{-1}\end{array}\right) \mid a \in k^{*}, b \in k\right\}$.

6 -n. (for some $n<\infty)$ : One dimensional abelian compact ( $k$-anisotropic) tori.The number of these tori can be shown to be bounded by $\left[k^{*}: k^{* 2}\right]^{2}$. When $k=\mathbb{C}$ there is no such torus and for $k=\mathbb{R}$ there is (up to conjugation) exactly one such torus, namely, $S O(2)$.

The classification of this lemma is routine but tedious. We omit the details. We shall also need the following two facts (for proofs see [41, A.5 A.6 A.7] and references therein): 
4.12 Theorem. Suppose $G$ acts smoothly on $S$ (2.1). Then there exists a Borel section for the action, i.e., there is a Borel subset which meets every $G$-orbit exactly once.

4.13 Theorem. Suppose $X, Y$ are standard Borel spaces (i.e. isomorphic to a Borel subset of a complete separable space). If $f: X \rightarrow Y$ is a surjective Borel map which is countable to one then there exists a Borel section $f^{-1}: Y \rightarrow X$.

As explained after 4.10, Theorem 1.6 will be established once we prove:

4.14 Theorem. If $\left(S L_{2}, V, \mu\right)$ is an additive $S L_{2}$-system, then there is a fixed point in $V$ (for the action of $S L_{2}$ ).

Proof. The strategy here is to assume that no such fixed point exists, to divide $V_{k}$ into finitely many Borel subsets, according to 4.11 and stability groups of points, and finally to deduce from the fact that one of these subsets gets positive measure that a corresponding homogeneous $S L_{2}(k)$ space has an invariant charge, contradicting 4.10 .

To ease notation, we shall omit the $k$-subscript and always work with the $k$ points of our varieties. Thus $V=V_{k}$ and $G=S L_{2}(k)$ acts on $V$, where by the contradicting assumption, all stability groups of points are conjugated in $S L_{2}(k)$ to one of the $H_{i}, 1 \leq i \leq n$, in correspondence to 4.11 .

For $1 \leq i \leq n$ define $V_{i}=\left\{v \in V \mid\right.$ Stab $\left.v=H_{i}\right\}$ (where for $i=1,2$ we mean that $S t a b v$ belongs to the family of subgroups described there). It is easy to verify that every $V_{i}$, as well as $G V_{i}=\left\{g v \mid g \in G, v \in V_{i}\right\}$ is a Borel subset of $V$, and that $V=\bigcup_{i=1}^{n} G V_{i}$. Recall that by $2.4 G$ and all its algebraic subgroups act smoothly on $V$, and thus on any $G V_{i}$. By 4.12 there is a Borel section for this action.

Since $\mu(V)=1$ there exists $i$ such that $\mu\left(G V_{i}\right)>0$ and we deal first with the case $3 \leq i \leq n$ : Define $N_{i}=$ Normalizer $_{G} H_{i}$, and observe that $N_{i}$ acts (smoothly) on $V_{i}$. Let $A_{i}$ be a Borel section for this action and define $f: G / H_{i} \times A_{i} \rightarrow G V_{i}$ by $f(\bar{g}, a)=g a$ (well defined).

Claim. $f$ is one to one and onto.

Proof of the claim. The "onto" is obvious. To see that $f$ is one to one assume that $\bar{g}_{1} a_{1}=\bar{g}_{2} a_{2}$. Choosing representatives we get $g_{1} a_{1}=g_{2} a_{2} \Rightarrow g_{2}^{-1} g_{1} a_{1}=a_{2}$ and denoting $g=g_{2}^{-1} g_{1}$ we get that $g a_{1}=a_{2}$ and need to show that $g \in H_{i}$ and $a_{1}=a_{2}$.

Indeed, Stab $a_{1}=H_{i}=S t a b a_{2}=g H_{i} g^{-1} \Rightarrow g \in N_{i} \Rightarrow a_{1}, a_{2}$ are in the same $N_{i}$ orbit $\Rightarrow a_{1}=a_{2}=a \Rightarrow g a=a \Rightarrow g \in H_{i}$ and the claim follows.

Let $\varphi=f^{-1}: G V_{i} \rightarrow G / H_{i} \times A_{i}$ be the inverse Borel map (4.13) and $\pi=$ projection from $G / H_{i} \times A_{i}$ to $G / H_{i}$. Then $\pi \circ \varphi: G V_{i} \rightarrow G / H_{i}$ is clearly a Borel $G$-equivariant map and therefore $\pi \circ \varphi_{*}\left(\left.\mu\right|_{G V_{i}}\right)$ is a $G$-invariant charge on $G / H_{i}$ (recall that if $\psi: X \rightarrow Y$ is a Borel map, and $\mu$ is a charge on $X$, then $\psi_{*} \mu$ is the charge on $Y$ defined by $\left.\psi_{*} \mu(A)=\mu\left(\psi^{-1}(A)\right)\right)$. As $H_{i}$ is amenable this contradicts 4.10 .

We are thus left with the cases $i=1,2$. The problem here is that one has to deal with infinitely many groups together. Let us check the case $i=2$, i.e., we assume that $\mu\left(G V_{2}\right)>0$.

The Borel subgroup $B=\left(\begin{array}{cc}\beta & \alpha \\ 0 & \beta^{-1}\end{array}\right)$ normalizes each of the groups $\left(\begin{array}{cc}\lambda & \alpha \\ 0 & \lambda^{-1}\end{array}\right)$ and therefore it acts on $V_{2}$. Let $A \subseteq V_{2}$ be a Borel section for this action. Define 
$N=\left\{\left(\begin{array}{ll}1 & t \\ 0 & 1\end{array}\right)\right\}$ and $f: G / N \times A \rightarrow G V_{2}$ by $f(\bar{g}, a)=g a$. Again $f$ is well defined and we now have:

Claim. $f$ is onto and finite to one.

Proof of the claim. The "onto" is obvious. To verify that $f$ is finite to one suppose $\bar{g}_{1} a_{1}=\bar{g}_{2} a_{2}$, fix representatives: $g_{1} a_{1}=g_{2} a_{2}$ so $g a_{1}=a_{2}\left(g=g_{2}^{-1} g_{1}\right)$ and we now show that $a_{1}=a_{2}$ and $\bar{g}_{\bmod N}=\left(\begin{array}{cc}\lambda & 0 \\ 0 & \lambda^{-1}\end{array}\right)$ (where $\lambda$ is a root of unity depending only on $a_{1}=a_{2}$, thus takes only finitely many values).

Denote $S_{i}=\operatorname{Stab}_{i}(i=1,2)$ and from $g a_{1}=a_{2}$ it follows that $g S_{1} g^{-1}=S_{2}$. In particular $g B g^{-1} \cap B \supseteq\left(\begin{array}{ll}1 & t \\ 0 & 1\end{array}\right)$ and this implies that $g \in B$. But if $a_{1}, a_{2}$ are in the same $B$-orbit $a_{1}=a_{2}=a$, so $g a=a$. Knowing the stabilizers of points in $A$ we deduce that $g \in\left(\begin{array}{cc}\lambda & \alpha \\ 0 & \lambda^{-1}\end{array}\right)$ or $\bar{g}_{\bmod N}=\left(\begin{array}{cc}\lambda & 0 \\ 0 & \lambda^{-1}\end{array}\right)$ as required in the claim.

By 4.13 there exists a Borel section $\varphi=f^{-1}: G V_{2} \rightarrow G / N \times A$. Let $\pi$ be the projection from $G / N \times A$ to $G / N . \pi \circ \varphi: G V_{2} \rightarrow G / N$ is a Borel map which is now not necessarily $G$-equivariant, however the proof of the claim shows that if $\varphi(v)=\left(\bar{g}_{1}, a\right)$ then $\left.\varphi(g v)=\overline{\left(g g_{1}\left(\begin{array}{cc}\lambda & 0 \\ 0 & \lambda^{-1}\end{array}\right)\right.}, a\right)$. Thus, by further projecting from $G / N$ to $G / B$ we get a $G$-equivariant map: $G V_{2} \rightarrow G / N \times A \rightarrow G / N \rightarrow G / B$. As in the first case we arrive at a contradiction by pushing the invariant charge, applying 4.10 and the amenability of $B$.

We are left with the case $i=1$ which is dealt with in a similar manner as was just shown. Here one works with a maximal compact $\operatorname{subgroup} C$ which contains all (conjugates of) the finite stabilizers. We only remark that although this subgroup need not be algebraic (this happens only when $k=\mathbb{R}$ ), every action of a compact group is smooth [41, 2.1.21], and thus a $G$-invariant charge on $G / C$ is obtained, contradicting the amenability of $C$ and 4.10. This completes the proof of 4.14 and with it the proof of Theorem 1.6.

Remark. Notice that in the proof of Theorem 4.14 the algebraic structure was actually used only for two features of the action: the smoothness and the fact that every stabilizer is algebraic. We claim that this can be used to show that the final statement in Theorem 1.6, namely, that invariant charges must be supported on fixed points, holds for every action of a disamenable group $G_{k}$, possessing these two features. To prove this we first notice that, as in 4.9 , by $k$-disamenability all the copies of $S L_{2}(k)$ in $G_{k}$ generate a Zariski dense subgroup and hence, by dimension argument, there are finitely many of them which already have this property. For each one of those, by (the proof of) 4.14 the fixed points have full measure so the intersection of the fixed point sets also has full measure, and every point there is fixed by all our $S L_{2}(k)$. As stabilizers are algebraic, every such point must be fixed by $G_{k}$, which proves our claim. We shall use this remark later but also note here that there are several non-algebraic actions for which the verification of these two features is significant in the course of the proof of the super-rigidity theorem (see $[41,3.2 .17-3.2 .18,3.3 .1,3.2 .2])$.

The following corollary will be of importance in the next section: 
4.15 Corollary. Let $V$ be the full $n$-dimensional affine space and $G<G L_{n}=$ $G L(V)$ be a k-group which acts irreducibly on $V$. If $G_{k}$ is not amenable, then there is no invariant charge for its action on $V_{k}-\{0\}=k^{n}-\{0\}$.

Proof. We assume that $\mu$ is an invariant charge and prove that $G_{k}$ is amenable by showing that $N$, the $k$-disamenable radical of $G$, is trivial (this will suffice due to 4.4). By Theorem 1.6, $\mu(\text { FixN })_{k}=1$, so letting $W=F i x N$ we have that $W \subseteq V$ is a $G$-invariant subspace with $\operatorname{dim} W>0$. By irreducibility of the $G$-representation $W=V$, so $N$ acts trivially on $V$ and the proof is complete.

Clearly if $G$ is $k$-disamenable it is $k$-discompact and by 3.13 its radical can be triangulized over $k$. Furthermore, we shall need the following:

4.16 Lemma. If $G$ is $k$-disamenable then its radical identifies with its unipotent radical. In particular, if $G$ is also not semisimple then $Z\left(\operatorname{Rad} G_{k}\right) \cong k^{n}$, with $n>0$.

Proof. Let $R_{u}$ be the unipotent radical of $R$ - the radical of $G . \quad R_{u} \triangleleft G$ and we need to show that $R_{u}=R$. $G / R_{u}$ is $k$-disamenable and reductive. However such a group has to be semisimple since its radical is $k$-isomorphic to the quotient of the group by its maximal semisimple subgroup.

We now turn back to discuss Corollary 1.7.

4.17 Definition. Let $G$ be a l.c. group and $\Gamma<G$ a closed subgroup. $\Gamma$ is said to be co-amenable in $G$ if there is a $G$-invariant charge on $G / \Gamma$.

Corollary 1.7 thus asserts that if $G$ is $k$-disamenable and $\Gamma<G_{k}$ is co-amenable, then $\Gamma$ is Zariski dense. In [12] a comprehensive analysis of co-amenability was carried out by Eymard. It is shown there that most of the known equivalent characterizations of amenable groups pass to the relative co-amenable situation. As we shall have some occasions to use it, we formulate the following:

4.18 Theorem (Eymard). Let $\Gamma<G$ be a closed subgroup of a l.c. group $G$. Then the following conditions are equivalent:

1. $\Gamma$ is co-amenable in $G$.

2. There is a $G$-invariant mean (i.e. a positive linear functional) on $L^{\infty}(G / \Gamma)$.

3. The quasi-regular representation of $G$ on $L^{2}(G / \Gamma)$ weakly contains the trivial representation (see Definition 5.1).

4. If $G$ acts continuously on a compact space $X$ and there is a point in $X$ fixed by

$\Gamma$, then there is a $G$-invariant $\sigma$-additive probability measure on $X$.

5. If $G$ acts continuously and affinely on a compact convex set $Q$ and there is a point in $Q$ fixed by $\Gamma$, then there is a point in $Q$ fixed by $G$.

Moreover, $G$ is amenable iff $\{e\}$ is co-amenable in $G$ and, more generally, if $\Gamma$ is amenable and co-amenable in $G$ then $G$ is amenable.

Clearly, if $H \triangleleft \Gamma$ then $H$ is co-amenable in $\Gamma$ iff $\Gamma / H$ is an amenable group. Furthermore, if $\Gamma$ is a lattice in $G$ it is co-amenable in it, and using condition 5, we see that if $H<\Gamma<G$, each co-amenable in the next, then $H$ is co-amenable in $G$. Therefore if $\Gamma$ is a lattice in $G$ and $H \triangleleft \Gamma$ is such that $\Gamma / H$ is amenable but not finite, then $H$ is a co-amenable subgroup of $G$ which is not a lattice. There are however, more general constructions of co-amenable subgroups, as indicated in $[21]$. 
In [35] the Zariski density of subgroups which have subexponential co-growth in semisimple $k$-groups was proved (see there for a precise definition). It is easy to see that these subgroups satisfy condition 3 of 4.18 and are therefore co-amenable. However, as remarked in [21], there are other examples of co-amenable subgroups (as there are amenable groups which do not have subexponential growth), see also [32]. It can also be shown that if $K \backslash G / \Gamma$ is a rank-1 locally symmetric space for which the geodesic flow on the unit tangent bundle is ergodic (with respect to the volume measure), then $\Gamma$ must be co-amenable in $G$ (and hence also Zariski dense, by 1.7). Note that if $G$ has property $(T)$, then by 3 of 4.18 every co-amenable subgroup is a lattice, so for almost $k$-simple groups the new density theorem can give new Zariski dense subgroups only if their $k$-rank is 1 .

Finally, we take the opportunity to recall the very natural (open) question of Eymard: If $H<\Gamma<G$ and $H$ is co-amenable in $G$, is $H$ co-amenable in $\Gamma$ ? This is the analogue of the (non-trivial) fact that a closed subgroup of an amenable group is amenable, and perhaps the only major unsettled point in this general theory of Eymard. It follows from Theorem 1.6 that the answer is affirmative when $H, \Gamma, G$ are algebraic, but this partial result is yet far from being satisfactory.

We close this section by presenting a completely different direction in which Theorem 1.6 may be used. First, let us note that the equivalence between conditions 2,3 in 4.18 is quite general. The following statement is proven in [16] when $m$ is invariant, and in [13] in general (the implication $1 \Rightarrow 2$ we need, essentially appears in the proof of 5.6 below).

4.19 Theorem. Suppose $(X, m)$ is a measure $G$-space where $m$ is a $\sigma$-additive quasi-invariant ( $\sigma$-finite) measure. Let $U_{X}$ be the quasi-regular natural representation of $G$ on $L^{2}(X, m)$. Then the following are equivalent:

1. $I \prec U_{X}$ (i.e. $U_{X}$ weakly contains the trivial representation, see Definition 5.1).

2. There is a $G$-invariant mean on $L^{\infty}(X, m)$.

A mean $\varphi$ on $L^{\infty}(X, m)$ defines a charge $\mu$ by $\mu(A)=\varphi\left(1_{A}\right)$, so Theorem 4.19 gives a general method to construct invariant charges in the presence of a quasi-invariant $\sigma$-additive measure. We thus obtain, using 4.19 , the following representation theoretic conclusion from Theorem 1.6:

4.20 Theorem. Let $G$ be a $k$-disamenable group. Denote by $\mathcal{F}$ the family of all $G_{k}$-unitary representations of the form $L^{2}\left(V_{k}, m\right)$, where the action of $G$ on $V$ is as in 1.6, and $m$ is a $G_{k}$-quasi-invariant measure on $V_{k}$ such that $m\left(F i x G_{k}\right)=0$. Then $\mathcal{F}$ is isolated, in the Fell topology, from the trivial representation I. Moreover, the same conclusion holds for the family $\mathcal{F}$ of all the representations of the form $L^{2}\left(G_{k} / \Gamma\right)$ where $\Gamma<G_{k}$ varies over all the closed subgroups of $G_{k}$ which are not Zariski dense in $G$. Consequently, the same conclusion holds for all representations which are induced from non-Zariski dense closed subgroups.

Proof. Suppose the first assertion fails. This, by definition, means that there is a sequence of $G_{k}$-actions: $\left(V_{k}^{(n)}, m^{(n)}\right), n=1,2, \ldots$, such that the direct sum $\bigoplus L^{2}\left(V_{k}^{(n)}, m^{(n)}\right)$ weakly contains $I$. Denoting by $(X, m)$ the (disjoint) sum of all the spaces $\left(V_{k}^{(n)}, m^{(n)}\right)$ we see that $I \prec U_{X}$ and hence, from 4.19 , there is a $G_{k^{-}}$ invariant mean $\varphi$ on $L^{\infty}(X, m)$. $X$ is not necessarily a variety, but the $G_{k^{-}}$-action on it is still smooth, stabilizers of all points are algebraic, and $m\left(F i x G_{k}\right)=0$. However, this and the remark proceeding 4.14 contradict the existence of $\varphi$. To prove the second assertion we again assume the contrary and using the same strategy obtain a 
$G_{k^{-}}$invariant mean $\varphi$ on $L^{\infty}(Y, m)$, where $(Y, m)=\bigcup_{n}\left(G_{k} / \Gamma^{(n)}, m^{(n)}\right)$. Denoting by $H^{(n)}$ the Zariski closures of $\Gamma^{(n)}$ we have $G_{k^{-}}$(natural) maps $G_{k} / \Gamma^{(n)} \rightarrow G_{k} / H_{k}^{(n)}$ which together induce a $G_{k}$-map from $Y$ to $X=\bigcup_{n} G_{k} / H_{k}^{(n)}$. Projecting the mean $\varphi$ brings us to the situation of the first statement, and again, to a contradiction.

In [13] it is shown how the fact that $I \nprec L^{2}(X, m)$ may be used to obtain results on the "positive drift" of a $G$-random walk on $X$. The positivity of the Lyapunov exponent for random products of matrices is a special case of this phenomenon.

\section{KAZHDAN'S PROPERTY $(T)$ AND FINITELY ADDITIVE MEASURES}

In this section a strong connection is established between invariant charges and property $(T)$ of Kazhdan. The results of Section 4 are thus applied to investigate property $(T)$. We first recall some of the basic definitions:

5.1 Definition. (i) Suppose $(\pi, \mathcal{H})$ is a (continuous) unitary representation of a l.c. group $G$. We say that $\pi$ weakly contains the trivial representation $I$ (denoted $I \prec \pi$ ) if for every compact set $K \subseteq G$ and $\varepsilon>0$ there exists $v \in \mathcal{H},\|v\|=1$, such that $\forall g \in K: \quad\|\pi(g) v-v\| \leq \varepsilon \quad$ (such a vector is referred to as $(K, \varepsilon)$ invariant).

(ii) $G$ has property (T) (of Kazhdan) if whenever $I \prec \pi$ then necessarily $I \subseteq \pi$.

Property $(T)$ for a l.c. group $G$ is easily seen to be equivalent to the existence of a compact set $K \subseteq G$ and $\varepsilon>0$ such that every unitary representation of $G$ containing a $(K, \varepsilon)$ invariant vector contains an invariant vector. Strengthening this property we shall discuss:

5.2 Definition. The l.c. group $G$ is said to have strong property (T) (abbreviated notation: st.pr.(T)), if there exists a finite set $F \subseteq G$ and $\varepsilon>0$ such that whenever a unitary $G$-representation $\pi$ has an $(F, \varepsilon)$ invariant vector then $I \subseteq \pi$.

The following assertion is easily verified:

5.3 Lemma. If $H \triangleleft G$ and both $H$ and $G / H$ have st.pr.( $T)$, then so does $G$. If $\varphi: G \rightarrow H$ is a homomorphism with dense image, and $G$ has st.pr.(T), then so does $H$.

If $G$ is discrete, obviously the strong and ordinary property $(T)$ coincide. Every compact group has property $(T)$, however $S^{1}=\mathbb{R} / \mathbb{Z}$ does not have st.pr.(T). Indeed, for every finite set $F \subseteq S^{1}$ and $\varepsilon>0$ there exists $0 \neq n \in \mathbb{Z}$ such that the character $e^{2 \pi i n x}$ is $(F, \varepsilon)$ invariant. More generally, it can be shown that if $G$ is amenable as a discrete group, then it has st.pr.(T) only if it is finite. Applying 5.3 and the above observation we see that if a connected group $G$ has some non-trivial character, then $G$ cannot have st.pr.(T). In 5.18 we will prove that, at least for connected Lie groups, this is the only obstruction standing between the ordinary property $(T)$ and the stronger one.

In an analogous way we strengthen the notion of relative property $(T)$ (whose original definition we shall not need in the sequel, see e.g. [17]):

5.4 Definition. Suppose $A$ is a l.c. abelian group and that $G$ is a l.c. group acting by automorphisms on $A$. We say that the pair $(G, A)$ has strong relative property (T) (abbreviated: st.r.pr.(T)), if there exists a finite set $F \subseteq G$ and $\varepsilon>0$ with the following property: 
If $\pi$ is a unitary representation of the semi-direct product $G \ltimes A$ for which $\left.\pi\right|_{G}$ contains an $(F, \varepsilon)$ invariant vector, then $\left.I \subseteq \pi\right|_{A}$.

Recall that if $G$ acts on $A$ as above, then $G$ acts on its dual group of characters$\hat{A}$, by $g \chi=\chi \circ g^{-1}$. Obviously, 0 , the trivial element of $\hat{A}$, is fixed by this action.

5.5 Theorem. Suppose $G, A$ are as above and that there is no invariant charge for the dual action of $G$ on $\hat{A}-\{0\}$. Then $(G, A)$ has st.r.pr.(T).

This establishes a connection between charges and property $(T)$ which, together with the results of Section 4, will enable us to study this property. We show in 5.7 that the converse to 5.5 also holds in some cases. For the proof of 5.5 we shall use the following:

5.6 Lemma. Suppose $G$ acts by Borel maps on a Borel space $X$. If for every finite set of measurable bounded real functions $Q=\left\{f_{1} \ldots f_{n}\right\}$, any set $F=\left\{g_{1} \ldots g_{n}\right\} \subseteq$ $G$ and every $\varepsilon>0$, there is a charge $\mu$ on $X$ which is $(Q, F, \varepsilon)$ invariant, i.e.,

$$
\forall 1 \leq i \leq n:\left|\int f_{i}\left(g_{i} x\right) d \mu(x)-\int f_{i}(x) d \mu(x)\right| \leq \varepsilon .
$$

Then there is a $G$-invariant charge on $X$.

Proof of 5.6. Let $B(X)$ denote the Banach space of all measurable bounded real functions on $X$ with the sup norm. The space of all finitely additive measures (finite, but not necessarily positive) on $X$ may be identified via integration with the dual $B(X)^{*}$, and applying Alaoglu's theorem it follows that the set of charges on $B(X)$ is compact in the weak-* topology. For every $Q, F, \varepsilon$ as in 5.6 denote by $\mathcal{F}_{Q, F, \varepsilon}$ the set of all $(Q, F, \varepsilon)$ invariant charges (as defined there). Every $\mathcal{F}_{Q, F, \varepsilon}$ is clearly closed in the weak-* topology, non-empty by the assumption, and the family of these $\mathcal{F}_{Q, F, \varepsilon}$ is closed under finite intersections. By compactness the intersection of them all is not empty, giving the required charge.

Proof of 5.5. We generalize an idea of Furstenberg [17] (used in [8] to compute Kazhdan constants for $S L_{3}(\mathbb{Z})$ ). By the assumption and 5.6 there exist finite sets $F \subseteq G, Q \subseteq B(\hat{A}-\{0\})$ and $\varepsilon>0$ such that there is no $(Q, F, \varepsilon)$ invariant charge on $\hat{A}-\{0\}$. By changing $\varepsilon>0$ we may assume that $|f| \leq 1$ for all $f \in Q$. We will show that $\left(F, \frac{\varepsilon}{2}\right)$ is as required in Def. 5.4 for $(G, A)$.

Let $\pi$ be a unitary representation of $G \ltimes A$ and $P$ be the corresponding projection valued measure for $\left.\pi\right|_{A}$. Recall that this measure assigns to every Borel set $B \subseteq \hat{A}$ an orthogonal projection $P(B)$ of $\mathcal{H}$, and has the following properties:

1. $P(\{0\})=$ projection onto the subspace of $A$ invariant vectors.

2. For all unit vectors $v \in \mathcal{H}, B \rightarrow\langle P(B) v, v\rangle$ is a $\sigma$-additive probability measure on $\hat{A}$.

3. For every $g \in G$ and $B \subseteq \hat{A}: \quad P(g B)=\pi(g) P(B) \pi(g)^{-1}$.

We assume that $\left.\pi\right|_{A}$ has no invariant vectors and prove that there is no unit vector which is $\left(F, \frac{\varepsilon}{2}\right)$ invariant. The non-existence of $A$-invariant vectors and property 1 above imply that for every $v \in \mathcal{H}$ the measure given by 2 has no mass in 0 , and therefore may be considered as a charge on $\hat{A}-\{0\}$. Assuming that $\|v\|=1$ is $\left(F, \frac{\varepsilon}{2}\right)$ invariant, we get that the corresponding measure $\mu_{v}(B)=\langle P(B) v, v\rangle$ 
satisfies for all $g \in F$ :

$$
\begin{aligned}
&\left|\mu_{v}\left(g^{-1} B\right)-\mu_{v}(B)\right|=\left|\left\langle\pi\left(g^{-1}\right) P(B) \pi(g) v, v\right\rangle-\langle P(B) v, v\rangle\right| \\
& \leq\left|\left\langle\pi\left(g^{-1}\right) P(B) \pi(g) v, v\right\rangle-\left\langle\pi\left(g^{-1}\right) P(B) v, v\right\rangle\right| \\
&+\left|\left\langle\pi\left(g^{-1}\right) P(B) v, v\right\rangle-\langle P(B) v, v\rangle\right| \\
&=\left|\left\langle\pi\left(g^{-1}\right) P(B)(\pi(g) v-v), v\right\rangle\right|+|\langle P(B) v,(\pi(g) v-v)\rangle| \\
& \leq\left\|\pi(g)^{-1} P(B)\right\|\|\pi(g) v-v\|+\|P(B)\|\|\pi(g) v-v\| \leq \frac{\varepsilon}{2}+\frac{\varepsilon}{2}=\varepsilon .
\end{aligned}
$$

Approximating each $f \in Q$ uniformly by simple functions, recalling that $|f| \leq 1$ and that $\left|\mu_{v}\left(g^{-1} B\right)-\mu_{v}(B)\right| \leq \varepsilon$ for every Borel set $B \subseteq \hat{A}-\{0\}$, it follows that $\mu_{v}$ is $(F, Q, \varepsilon)$ invariant, which is a contradiction.

5.7 Theorem. The converse in 5.5 holds in either one of the following cases:

1. $G$ acts transitively on $\hat{A}-\{0\}\left(\right.$ e.g. $\left.G=S L_{n}(\mathbb{R}), A=\mathbb{R}^{n}\right)$.

2. $A$ is compact (e.g. $G=S L_{n}(\mathbb{Z}), A=\mathbb{R}^{n} / \mathbb{Z}^{n}$ ).

Proof. We assume that $(G, A)$ has st.r.pr.(T) and prove that there is no $G$-invariant charge on $\hat{A}-\{0\}$. We claim that in both cases, the existence of such a charge implies that there is a $G$-invariant mean on $L^{\infty}(\hat{A}-\{0\})$. In case 1 this follows from $4.18(1 \Rightarrow 2)$ and in case 2 this is clear as $\hat{A}$ is discrete so any charge may also be regarded as a mean on $L^{\infty}$. Applying 4.18 we get that the representation of $G$ on $L^{2}(\hat{A}-\{0\})$ weakly contains $I$. Notice that in case 1 the Haar measure of $\hat{A}$ may not be preserved by the action but rather multiplied by a $G$-character. This will not interfere with our arguments.

Let $f \rightarrow \hat{f}$ be the Fourier transform from $L^{2}(\hat{A})$ to $L^{2}(\hat{\hat{A}}) \cong L^{2}(A)$. This is an isometry which intertwines the $G$-action. By applying it to the "almost invariant functions" in $L^{2}(\hat{A}-\{0\})$ one gets, in case $A$ is compact, "almost invariant functions" in $L_{0}^{2}(A)=\left\{f \in L^{2}(A) \mid \int f d \mu=0\right\}$, and in case 1, in $L^{2}(A)$. However $G \ltimes A$ acts naturally on $A$ by $\left(g, a_{1}\right) a=g a+a_{1}$ and it follows from the assumption that $(G, A)$ has st.r.pr.(T), that there is an $A$-invariant function in this representation, a contradiction in both cases.

Notice that when $A$ is discrete neither the assumption nor the conclusion of 5.5 holds. However the weaker property, that every representation of $G \ltimes A$ which weakly contains $I$ (as a representation of the whole group) contains an $A$-invariant vector, may hold, as in the case of $G=S L_{2}(\mathbb{Z}), A=\mathbb{Z}^{2}$. This is usually proven by inducing the representation (in the above example, to $S L_{2}(\mathbb{R}) \ltimes \mathbb{R}^{2}$ ), but using refinements of some of the ideas presented here, a direct proof of it can be given.

We would like to discuss a little further the case where $A$ is compact, starting with the following result of Rosenblatt [30] (the implication $\Rightarrow$ ) and Schmidt [34] (the implication $\Leftarrow$ ), which is in the spirit of 4.19:

5.8 Theorem (Rosenblatt-Schmidt). If $G$ is countable and acts by measure preserving transformations on $(X, \mu)$ (where $\mu$ is a $\sigma$-additive probability measure), then $I \nprec L_{0}^{2}(X)$ as $G$-representations $\Leftrightarrow \mu$ integration is the unique $G$-invariant mean on $L^{\infty}(X, \mu)$.

Combining 4.19, 5.5, 5.7, 5.8 and the use of the Fourier transform as in 5.7 yields:

5.9 Theorem. Suppose that $A$ is a compact abelian group and $G$ is a (countable) group of automorphisms of $A$. Then the following are equivalent: 
1. $(G, A)$ has st.r.pr. $(T)$.

2. The Haar integral on $A$ is the unique $G$-invariant mean on $L^{\infty}(A)$.

3. $I \nprec L_{0}^{2}(A)$ as $G$-representations.

4. There is no invariant charge for the action of $G$ on $\hat{A}-\{0\}$.

5. $I \nprec L^{2}(\hat{A}-\{0\})$ as $G$-representations.

Let us demonstrate briefly how some of the ideas presented in this paper come together in a short proof of the following theorem of Rosenblatt [30] (for $n \geq 3$ this was observed also by Margulis [25]).

5.10 Theorem. When $A=\mathbb{T}^{n}=\mathbb{R}^{n} / \mathbb{Z}^{n}$ and $G=S L_{n}(\mathbb{Z})$, condition 4 and hence all the other conditions in 5.9 are satisfied.

Proof. Assume the existence of an $S L_{n}(\mathbb{Z})$ invariant charge on $\mathbb{Z}^{n}-\{0\} \cong \hat{\mathbb{T}}^{n}-\{0\}$. Push the charge to the projective image of $\mathbb{Z}^{n}-\{0\}, \mathbb{P}^{n-1}(Q)$, to get an invariant $P S L_{n}(\mathbb{Z})$ charge there. It is not difficult to check that the action of $P S L_{n}(\mathbb{Z})$ on $\mathbb{P}^{n-1}(Q)$ is transitive so if $H<P S L_{n}(\mathbb{Z})$ is a stabilizer of any point in $\mathbb{P}^{n-1}(Q)$ we get that $H$ is co-amenable in $P S L_{n}(\mathbb{Z}) . P S L_{n}(\mathbb{Z})$, being a lattice in $P S L_{n}(\mathbb{R})$, is co-amenable so by the transitivity of this relation (see the discussion proceeding 4.18) $H$ is co-amenable in $P S L_{n}(\mathbb{R})$. However it is easy to see that $H$ is not Zariski dense in $P S L_{n}(\mathbb{R})$ and this contradicts 1.7 .

We remark that condition 3 in 5.9 may be used to obtain exponential rate of mixing and fast rate of convergence in a mean and pointwise ergodic theorem along random products of automorphisms, so 5.10 has purely ergodic theoretic implicationssee [14] for details.

We now turn back to the main discussion.

5.11 Theorem. Let $G$ be a $k$-disamenable group. If $G_{k} / R a d G_{k}$ has property $(T)$, then so does $G_{k}$.

In the proof we shall use the following, due to Serre [17, p. 28]:

5.12 Proposition. Let $G$ be a l.c. $\sigma$-compact group. Suppose that $C<Z(G)$, and that $G /[G, G]$ is compact. If $G / C$ has property $(T)$, then so does $G$.

Proof of 5.11. The proof goes by induction on $d=\operatorname{dim}(\operatorname{Rad} G)$. When $d=0$ our claim is just the assumption. If $d>0$, by 4.16 we have $Z\left(\operatorname{Rad} G_{k}\right) \cong k^{n}$ with $n>0$, and denote this (non-trivial) vector group by $V$. Clearly $V \triangleleft G_{k}$ and the conjugation action of $G_{k}$ on $V$ is an automorphism action, i.e., an action by $(k-)$ linear transformations. Let $A \subseteq V$ be an irreducible (non-trivial) $G_{k}$-subspace. $A \triangleleft G_{k}$ and there are two possibilities:

1. $\operatorname{dim}_{k} A=1$. Then $G_{k}$ acts by characters, but since the action is algebraic and $G$ is $k$-disamenable the action is trivial, so $A \subseteq Z\left(G_{k}\right)$. By the induction hypothesis, $G_{k} / A$ (having the same semisimple image and a radical of lower dimension) has property $(T)$. As $G$ is $k$-disamenable, $G_{k} /\left[G_{k}, G_{k}\right]$ is trivial, so by $5.12 G_{k}$ has property $(T)$.

2. $\operatorname{dim}_{k} A>1$. Recall that if $k$ is a l.c. field then $\hat{k}^{n} \cong k^{n}$ (not canonically), and the dual action of a $k$-linear transformation is by $g \mapsto\left(g^{t}\right)^{-1}$. Therefore starting with an algebraic action of $G_{k}$ on $A$ we get an algebraic action on $\hat{A}$. Observe that the action of $G_{k}$ on $\hat{A}$ is irreducible as well: if $W \subseteq k^{n}$ is an invariant subspace for $H \subseteq G L_{n}(k)$ then $W^{\perp}$ (orthogonal with regards to the standard bi-linear form) is invariant under the action of $H$ via $h \mapsto\left(h^{t}\right)^{-1}$. By the $k$-disamenability of 
$G$, the image of $G_{k}$ in $G L(\hat{A})$ is not amenable and as the action is irreducible, we deduce from 4.15 and 5.5 that $(G, A)$ has st.r.pr. (T). Now, let $(\pi, \mathcal{H})$ be a representation of $G_{k}$ such that $I \prec \pi$. Define a representation $\pi_{1}$ of $G_{k} \ltimes A$ by $\pi_{1}(g, a)=\pi(g a)$. By the assumption, $\left.I \prec \pi_{1}\right|_{G_{k}}$, hence $\left.I \subseteq \pi_{1}\right|_{A}$ and so $\left.I \subseteq \pi\right|_{A}$. Let $\mathcal{H}_{A}=\{v \in \mathcal{H} \mid \pi(a) v=v \forall a \in A\}$ and write $\mathcal{H}=\mathcal{H}_{A} \oplus \mathcal{H}_{A}^{\perp}$ an orthogonal sum of $G_{k}$-invariant subspaces $\left(A \triangleleft G_{k}\right)$. Since $I \prec \pi$ the restriction of $\pi$ to at least one of these subspaces weakly contains $I$. It cannot be $\mathcal{H}_{A}^{\perp}$ (as the last argument shows, we would then have an $A$-invariant non-zero vector in $\mathcal{H}_{A}^{\perp}$ ) so it must be $\mathcal{H}_{A}$. The representation of $G_{k}$ on $\mathcal{H}_{A}$ weakly contains $I$ and factors through $G_{k} / A$ which, by induction hypothesis, has property $(T)$. Therefore there is a $G_{k}$-invariant vector in $\mathcal{H}_{A}$, as required.

We now note the modifications needed to prove the following strengthening of 5.11:

5.13 Theorem. If $G$ is $k$-disamenable and $G_{k} /$ Rad $G_{k}$ has property $(T)$, then $G_{k}$ has st.pr.(T).

Proof. The proof of 5.11 carries through with minor changes. The main tool there is the st.r.pr.( $\mathrm{T}$ ) which was stated and proved in this stronger form (in 5.5) exactly for this reason. Serre's proposition (5.12) is valid if we replace property $(T)$ by st.pr.(T) in both the assumption and the conclusion, with the slight change that we now have to assume that $G /[G, G]$ is finite rather than compact.

The last modification needed is in the base of the induction, since we now claim a non-trivial result even for semisimple groups. In light of 5.3 we have to show that if $G$ is almost $k$-simple and $G_{k}$ is non-compact and has property $(T)$ then $G_{k}$ has st.pr.(T). (All the factors appearing in $G_{k}$ are non-compact since $G$ is $k$ disamenable.) It is possible to use the strength of 5.5 and imitate the well known argument to get this result when $k$-rank $G>1$. However, we would then be left with the exceptions: $S p(n, 1), F_{4}$. To deal also with them, one can argue as follows: $G_{k}$ always contains a lattice (see [5]) $\Gamma$, which has property $(T)$ if $G_{k}$ has it. Therefore there exist $F \subseteq \Gamma$ and $\varepsilon>0$ such that every unitary representation of $\Gamma$ containing an $(F, \varepsilon)$ invariant vector has an invariant vector. By Howe-Moore's theorem over local fields this $(F, \varepsilon)$ "almost" works for $G_{k}$, as every $\Gamma$-invariant vector is invariant under $G_{k}^{+}$which is of finite index in $G_{k}$ (see $[41,10.1 .3,10.1 .4,10.1 .2]$ ). To fix this simply throw into $F$ a finite number of representatives of $G_{k} / G_{k}^{+}$and this completes the proof.

We can now easily prove Theorem 1.9 (stated in the introduction):

5.14 Proof of 1.9. The necessity of the first condition is obvious and that of the second follows from the fact that $G_{k} / N_{k}$ is always amenable, so if $G_{k}$ has property $(T)$ then $G_{k} / N_{k}$ also has it, and thus it must be compact. Conversely, as $N \triangleleft$ $G, N / \operatorname{Rad} N$ is normal in $G / \operatorname{Rad} G$, so $N_{k} / \operatorname{Rad} N_{k}$, being (locally) isomorphic to a product of some of the simple components of $G_{k} / \operatorname{Rad} G_{k}$ (in fact, the non-compact ones) has property $(T)$. Applying 5.11 we deduce that $N_{k}$ has property $(T)$, and since $G_{k} / N_{k}$ is compact, so does $G_{k}$.

We now discuss st.pr.(T) for compact groups. While in the context of ordinary property $(T)$ they form the (only) trivial examples of Kazhdan groups, st.pr.(T) for the compact (non-finite) groups seems to be extremely interesting. It turns out that much of the work here was done under the theme of uniqueness of the 
Haar measure as an invariant charge for the self-multiplication action of the group. Before elaborating on that we observe a pleasant feature of compact groups:

5.15 Lemma. Let $G$ be a compact group. $G$ has st.pr.(T) iff there is a finite set $F \subseteq G$ and $\varepsilon>0$ such that $L_{0}^{2}(G)$ does not contain an $(F, \varepsilon)$ invariant vector. Equivalently, there exists a finitely generated subgroup $\Gamma<G$ such that as $\Gamma$-representations, $I \nprec L_{0}^{2}(G)$.

Proof. The necessity is obvious, for sufficiency use the Peter-Weyl theorem.

In the literature, st.pr.(T) for compact groups is also referred to as (not possessing) the mean zero weak containment property. Applying 5.15 and 5.8 one readily verifies:

5.16 Proposition. Let $G$ be a compact group. If $G$ has st.pr. $(T)$ then the Haar integral on $G$ is the unique $G$-invariant mean on $L^{\infty}(G)$.

Recall that the Ruziewicz problem, which essentially discusses such a uniqueness property, has been solved in the early 80 's using 5.16 . In all cases deep tools were applied: all the simple, compact Lie groups which are not locally isomorphic to $S O(n)$ for $n=2,3,4$, were shown by Margulis [25] to contain a finitely generated dense subgroup with property ( $T$ ) (for $S O(n) n \geq 5$, this was done simultaneously by Sullivan [36]). For $S O(n) n=3,4$, the Ramanujan conjecture (proved by Deligne) was applied by Drinfeld [11] (see also [24]) to construct an appropriate $\Gamma<G$, as in 5.15. Subsequently we have:

$\mathbf{5 . 1 7}$ Theorem. If $G$ is a simple, connected, non-abelian, compact Lie group then G has st.pr.(T).

See [23] for details and a comprehensive exposition of this subject. The following problem, however, suggests itself:

Question. Does the converse of 5.16 hold?

The difficulty arises due to the fact that a mean on $L^{\infty}(G)$ which is preserved by an action of a dense subgroup need not be $G$-invariant (such an example is presented in [31]).

When $G$ is totally disconnected, st.pr.(T) is also of interest. As shown in [31], this property is equivalent to the fact that the finite quotients of $G$ (by its open subgroups) can be made Cayley graph expanders. For instance, if $\hat{\mathbb{Z}}$ is the ring of adelic integers, then $S L_{3}(\hat{\mathbb{Z}})$ has st.pr.(T) (using the dense embedding of $S L_{3}(\mathbb{Z})$ ). From [42] it follows that $S L_{2}(\hat{\mathbb{Z}})$ does not contain a dense Kazhdan subgroup, however from Selberg's well known eigenvalue theorem: $\lambda_{1} \geq 3 / 16$, it follows that it has st.pr.(T) (in fact, a qualitative Selberg's theorem $\lambda_{1} \geq c>0$ is equivalent to the uniqueness of the Haar measure of $S L_{2}(\hat{\mathbb{Z}})$ as an $S L_{2}(\mathbb{Z})$-invariant finitely additive measure - see [31] for details and applications of these ideas). We shall not discuss further the disconnected case but rather return to show the following strengthening of Wang's result [38]:

5.18 Theorem. Let $G$ be a connected Lie group and $M \triangleleft G$ the smallest closed connected normal subgroup of $G$ containing all maximal non-compact simple subgroups. Then $G$ has property (T) iff $G / \operatorname{Rad} G$ has it and $G / M$ is compact. Furthermore, $G$ has st.pr.(T) iff in addition $G / M$ is semisimple. In particular, if $G$ has property (T) then it has st.pr.(T) if (and only if) it has no non-trivial characters. 
Proof. Case I. $G=M$ and $G=\left(\mathbf{G}_{\mathbb{R}}\right)_{0}(\mathbf{G}$ an $\mathbb{R}$-group $)$. By the statement at the end of $4.6 \mathbf{G}$ is $\mathbb{R}$-disamenable so by $5.13, G$ has st.pr.(T).

Case II. $G=M$. Notice that $[G, G]=G$. Let $A d: G \rightarrow \mathrm{GL}(\mathfrak{g})$ be the adjoint representation, and $H=A d G$, the image of $G . H$ is a linear Lie subgroup of $\operatorname{GL}(\mathfrak{g})$ which a priori is not even closed. However, since $[\mathfrak{g}, \mathfrak{g}]=\mathfrak{g}$ also , $[\mathfrak{h}, \mathfrak{h}]=\mathfrak{h}$ $(\mathfrak{h}=$ Lie $H)$, so $\mathfrak{h} \otimes \mathbb{C}$ is algebraic (cf. [4, 7.9]) which implies that $H=\left(\mathbf{H}_{\mathbb{R}}\right)_{0}$. Our assumptions on $G$ pass to $H$ and, using case I, $H$ has st.pr.(T). Since $H \cong G / Z(G)$ and $G=[G, G]$ it follows from the modification of 5.12 (discussed in 5.13) that $G$ has st.pr.(T).

General case. By case II it only remains to see that if $G / M$ is compact and semisimple it has st.pr.(T). (Notice that $M / \operatorname{Rad} M$ is normal in $G / \operatorname{Rad} G$ ). However this is exactly the contents of 5.17. Obviously if $G / M$ is just compact we have that $G$ has property (T).

The necessity of the conditions for the ordinary property $(T)$ is obvious (recall that $G / M$ is always amenable). To see their necessity for the stronger property notice that again if $G$ has st.pr.(T), so does $G / M$. As any compact Lie group is (locally) isomorphic to a product of simple groups (abelian and non-abelian), the projection onto any abelian factor must be finite (by 5.3 and the discussion thereafter). Finally, the last statement is obvious as the lack of any non-trivial character forces $G / M$ to be semisimple.

Closing a circle of ideas, we deduce from 5.8 and 5.18 the following generalization to the solution of the Ruziewicz problem:

5.19 Theorem. Let $G$ be a connected Kazhdan Lie group with no non-trivial characters. Suppose that $G$ acts by measure preserving transformations on a probability measure space $(X, \mu)$. Then $\mu$-integration is the unique $G$-invariant mean on $L^{\infty}(X, \mu)$.

This uniqueness of $\mu$ has ergodic theoretic implications (in the spirit mentioned after 5.10) also when $G$ is not discrete- see [14].

It is well known that every compact real matrix group is algebraic. This pleasant property enables us to deduce:

5.20 Theorem. If $H<G L_{n}(\mathbb{R})$ is any subgroup and $H$ has property $(T) /$ st.pr.(T) then so does (the $\mathbb{R}$-points of) its Zariski closure.

Proof. Denote $\mathbf{G}=\bar{H}$ and let $N \triangleleft \mathbf{G}$ be its $\mathbb{R}$-disamenable radical. By 5.18 it suffices to see that $\mathbf{G}_{\mathbb{R}} / \operatorname{Rad} \mathbf{G}_{\mathbb{R}}$ has property $(T)$ and that $\mathbf{G}_{\mathbb{R}} / N_{\mathbb{R}}$ is compact / compact and semisimple. The first condition follows by considering each of the simple factors of $G_{\mathbb{R}} / \operatorname{Rad} G_{\mathbb{R}}$ : If the projection of $H$ to it is not bounded then by Howe-Moore's theorem this simple factor must have property $(T)$, as does $H$. If the projection of $H$ is bounded, then by its Zariski density and the remark preceding 5.20, this factor is compact (and thus has property $(T)$ ). The second condition follows from the fact that $\mathbf{G}_{\mathbb{R}} / N_{\mathbb{R}}$ is always amenable and thus the closure of the image of $H$ in it is compact. Since this image is Zariski dense, as before, $G_{\mathbb{R}} / N_{\mathbb{R}}$ must be compact. In case $H$ has st.pr.(T), we need to show that $G_{\mathbb{R}} / N_{\mathbb{R}}$ has trivial radical. Indeed, since $H$ projects (algebraically) to the radical and its image is Zariski dense, again it is also topologically dense, so by 5.3 the radical has st.pr.(T), and hence it is trivial. 
The case of "ordinary" property $(T)$ in 5.20 is due to Wang [38]. A different strengthening of 5.20 is due to Zimmer [42]. The assumption $k=\mathbb{R}$ is necessary, and it may be interesting to compare 5.20 with [41, 3.2.22], from which an analogous statement can be deduced, replacing "property $(T)$ " by "amenable".

\section{REFERENCES}

[1] A' Campo, N. and Burger, M., Resaux arithmetiques et commensurateur d'apres G.A. Margulis, Invent. Math. 116 (1994), 1-25. MR 96a:22019

[2] Bernstein, I.N. and Zelevinskii, A.V., Representations of the group $G L(n, F)$ where $F$ is a non-archimedean local field, Russian Math. Surveys 31 (1976), 1-68. MR 54:12988

[3] Borel, A., Density properties for certain subgroups of semisimple groups without compact factors, Ann. Math. 72, (1960), 179-188. MR 23:A964

[4] Borel, A., Linear Algebraic Groups, W.A. Benjamin, New York, 1969. MR 40:4273

[5] Borel, A. and Harder, G., Existence of discrete co-compact subgroups of reductive groups over local fields, J. Reine Angew. Math. 298 53-64 (1978). MR 80b:22022

[6] Borel, A. and Serre, J.P., Theoremes de finitude en cohomologic galoisienne, Comm. Math. Helv. 39 (1964), 111-164. MR 31:5870

[7] Borel, A. and Tits, J., Groupes reductifs, Publ. Math. I.H.E.S. 27 55-150, (1965). MR 34:7527

[8] Burger, M., Kazhdan constants for $S L_{3}(\mathbb{Z})$, J. Reine Angew. Math, 423 36-67, (1991). MR 92c:22013

[9] Dani, S.G., A simple proof of Borel's density theorem, Math. Z. 174 81-94, (1980). MR 81m:22010

[10] Dani, S.G., On ergodic quasi-invariant measures of group automorphisms, Israel J. Math. 43 62-74 (1982). MR 85d:22017

[11] Drinfeld, V.G., Finitely additive measures on $S^{2}$ and $S^{3}$, invariant with respect to rotations, Func. Anal. and its Appl. 18 245-246 (1984). MR 86a:28021

[12] Eymard, P., Moyennes Invariantes et Representations Unitaires, Lecture Notes in Mathematics No. 300, Springer-Verlag 1972. MR 43:6740

[13] Furman, A. and Shalom, Y., Random walks in Hilbert spaces and Lyapunov exponents, in preparation.

[14] Furman, A. and Shalom Y., Sharp ergodic theorems for group actions and strong ergodicity, To appear in Ergodic Theory and Dynamical Systems.

[15] Furstenberg, H., A note on Borel's density theorem, Proc. AMS 55, 209-212 (1976). MR 54:10484

[16] Greenleaf, F.P., Amenable actions of locally compact groups, J. Funct. Anal. 4, 295-315, (1969). MR 40:268

[17] de la Harpe, P. and Valette, A., La Propriete (T) de Kazhdan pour les Groupes Localement Compacts, Asterisque vol. 175, Paris, Soc. Math. Fr. 1989. MR 90m:22001

[18] Helgason, S., Differential Geometry and Symmetric Spaces, Academic Press, New York, 1962. MR 26:2986

[19] Humphreys, J.E., Linear Algebraic Groups, Springer, New York, 1975. MR 53:633

[20] Iozzi, A., Invariant geometric structures: A non-linear extension of the Borel density theorem, Amer. J. Math. 114 627-648 (1992). MR 93k:22008

[21] Iozzi, A. and Nevo, A., Algebraic hulls and the Følner property, Geom. Funct. Anal. 6 (1996), 666-688. MR 97j:22011

[22] Kazhdan, D., Connection of the dual space of a group with the structure of its closed subgroups, Func. Anal. Appl. 1 63-65, (1967).

[23] Lubotzky, A., Discrete Groups, Expanding Graphs and Invariant Measures, Birkhäuser, Progress in Mathematics, 1994. MR 96g:22018

[24] Lubotzky, A., Phillips, R. and Sarnak, P., Hecke operators and distributing points on $S^{2}$, II, Comm. Pure and Applied Math. 39, 401-420, (1987). MR 88m:11025a

[25] Margulis, G.A., Some remarks on invariant means, Monat. fur Math. 90, 233-235, (1980). MR 82b:28034

[26] Margulis, G.A., Discrete Subgroups of Semisimple Lie Groups, Springer 1990. MR 92h:22021

[27] Mosak, R. and Moskowitz, M., Zariski density in Lie groups, Isr. J. Math. 52, 1-14, (1985). MR 87h:22010 
[28] Moskowitz, M., On the density theorem of Borel and Furstenberg, Ark. Math. 16 11-27, (1978). MR 58:22393

[29] Prasad, G., Elementary proof of a theorem of Tits and a theorem of Bruhat-Tits, Bull. Soc. Math. Fr. 110 197-202 (1982). MR 83m:20064

[30] Rosenblatt, J., Uniqueness of invariant means for measure preserving transformations, Trans. AMS 265, 623-636, (1981). MR 83a:28026

[31] Shalom, Y., Expanding graphs and invariant means, To appear in Combinatorica.

[32] Shalom, Y., Zariski closures of co-amenable subgroups and a spectral extension of "Tits' alternative", in preparation.

[33] Schmidt, K., Asymptotically invariant sequences and an action of $S L_{2}(\mathbb{Z})$ on the 2-sphere, Israel J. Math. 37, 193-208, (1980). MR 82e:28023a

[34] Schmidt, K., Amenability, Kazhdan's property (T), strong ergodicity and invariant means for ergodic group actions, Ergodic Theory and Dynamical Systems 1 223-236 (1981). MR 83m:43001

[35] Stuck, G., Growth of homogeneous spaces, density of discrete subgroups and Kazhdan's property (T), Invent. Math. 109 505-517, (1992). MR 93j:22022

[36] Sullivan, D., For $n>3$ there is only one finitely additive rotationally invariant measure on the n-sphere defined on all Lebesgue measurable subsets, Bull AMS 4, 121-123 (1981). MR 82b:28035

[37] Tits, J. Lectures on Algebraic Groups, Notes by P. Anche and D. Winter after a course of J. Tits 1966/7, Yale University.

[38] Wang, S.P., On the Mautner phenomenon and groups with property (T), Amer. J. Math. 104, 1191-1210, (1982). MR 84g:22033

[39] Wang, S.P., On anisotropic solvable linear algebraic groups, Proc. AMS 84, 11-15, (1982).

[40] Witte, D., Superrigidity of lattices in solvable Lie groups, Invent. Math. 122 (1995), no. 1, $147-193$.

[41] Zimmer, R.J., Ergodic Theory and Semisimple Groups, Boston: Birkhäuser, 1984. MR 86j:22014

[42] Zimmer, R.J., Kazhdan groups acting on compact manifolds, Invent. Math. 75, 425-436, (1984). MR 86a:22009

Institute of Mathematics, Hebrew University, Jerusalem 91904, Israel

Current address, September 98-September 99: Department of Mathematics, Princeton University, Princeton, New Jersey 08544-1000

E-mail address: yehuda@math.huji.ac.il 